\title{
Deconstructing the narrow-line region of the nearest obscured quasar
}

\author{
M. Villar Martín ${ }^{1,2}$, E. Bellocchi ${ }^{1}$, J. Stern $^{3}$, C. Ramos Almeida ${ }^{4,5}$, C. Tadhunter 6
}

R. González Delgado ${ }^{7}$

${ }^{1}$ Centro de Astrobiología (INTA-CSIC), Carretera de Ajalvir, km 4, E-28850 Torrejón de Ardoz, Madrid, Spain. villarmm@cab.inta-csic.es

${ }^{2}$ Astro-UAM, UAM, Unidad Asociada CSIC, Facultad de Ciencias, Campus de Cantoblanco, E-28049, Madrid, Spain

${ }^{3}$ Max Planck Institute für Astronomie, Königstuhl 17, D-69117 Heidelberg, Germany

${ }^{4}$ Instituto de Astrofísica de Canarias, C/ Vía Láctea, s/n, E38205 La Laguna,Tenerife, Spain

${ }^{5}$ Departamento de Astrofísica, Universidad de La Laguna, E-38206, La Laguna, Tenerife, Spain

${ }^{6}$ Department of Physics and Astronomy, University of Sheffield, Sheffield S3 7RH, UK

${ }^{7}$ Instituto de Astrofísica de Andalucía (CSIC), Glorieta de la Astronomía, s/n, 18008 Granada, Spain

\begin{abstract}
We study the physical and kinematic properties of the narrow line region (NLR) of the nearest obscured quasar MRK $477(z=0.037)$, using optical and near-infrared (NIR) spectroscopy. About 100 emission lines are identified in the optical+NIR spectrum (90 in the optical), including several narrow optical $\mathrm{Fe}^{+}$lines. To our knowledge, this is the first type 2 active galactic nucleus (AGN) with such a detection. The $\mathrm{Fe}^{+}$lines can be explained as the natural emission from the NLR photoionized by the AGN. Coronal line emission can only be confirmed in the NIR spectrum.

As in many other AGN, a significant correlation is found between the lines full width at half-maximum and the critical density $\log \left(n_{\text {crit }}\right)$. We propose that it is caused by the outflow. This could be the case in other AGNs.

The nuclear jet-induced ionized outflow has been kinematically isolated in many emission lines covering a broad range of ionization potentials and critical densities. It is concentrated within $R \sim$ few $\times 100$ pc from the central engine. The outflowing gas is denser $\left(n \gtrsim 8000 \mathrm{~cm}^{-3}\right)$ than the ambient non-perturbed gas $\left(n \sim 400-630 \mathrm{~cm}^{-3}\right)$. This could be due to the compression effect of the jet induced shocks. Alternatively, we propose that the outflow has been triggered by the jet at $R \lesssim 220$ pc (possibly at $\lesssim 30$ pc) and we trace how the impact weakens as it propagates outwards following the radiation-pressure dominated density gradient.

The different kinematic behaviour of [FeII $] \lambda 1.644 \mu \mathrm{m}$ suggests that its emission is enhanced by shocks induced by the nuclear outflow/jet and is preferentially emitted at a different, less reddened spatial location.
\end{abstract}

\section{Key words:}

galaxies: active quasars: emission lines - quasars: general - quasars: individual: MRK 477

\section{INTRODUCTION}

MRK 477 (SDSS J144038.1+533016, $z=0.037$ and luminosity distance $D_{L}=161 \mathrm{Mpc}$ ) is a type 2 luminous active galactic nucleus (AGN). Also known as I Zw 92, it was first identified by Zwicky (1966) as a compact galaxy. It is usually referred to as the most luminous Seyfert 2 in the local Universe. As pointed out by Heckman et al. (1997), it has the highest $[\mathrm{O}$ III $] \lambda 5007$ luminosity $\left(L_{[\mathrm{OIII}]}=3.3 \times 10^{42} \mathrm{erg}\right.$ $\mathrm{s}^{-1}$ ) of any of the 140 Seyfert nuclei (type 1 or 2) com- piled by Whittle (1992) and the fifth highest radio power $\left(P_{1.4 \mathrm{GHz}}=2.0 \times 10^{30} \mathrm{erg} \mathrm{s}^{-1} \mathrm{~Hz}^{-1}\right.$ at $\left.1.4 \mathrm{GHz}\right)$. Indeed, its high $L_{\text {[OIII] }}$ places it in the regime of optically selected obscured quasars (QSO2), according to the selection criteria defined by Zakamska et al. (2003),$L_{[\mathrm{OIII}]}>1.2 \times 10^{42} \mathrm{erg}$ $\mathrm{s}^{-1}$. The [OIII] luminosity implies a bolometric luminosity $L_{b o l}=8.6 \times 10^{45} \mathrm{erg} \mathrm{s}^{-1}$ (Stern \& Laor 2012; see also Lamastra et al. 2009), which is in the quasar range (Shen et al. 2011). 
The quasar host galaxy is interacting with an emission line companion, possibly a LINER, located 50 arcsec $(\sim 36$ $\mathrm{kpc}$ ) to the north (De Robertis et al. 1987). Hubble Space Telescope (HST) images are shown in Fig. 11 Both galaxies are connected by a faint bridge and prominent tidal tails are apparent. The ratio of stellar masses between the quasar host and the companion is M1/M2=1.6 (Koss et al. 2012).

The quasar host shows a compact blue central source, peculiar among type 2 AGNs, but no evidence for a broad line region (BLR), prompting some authors to suggest that the quasar lacks one. However, polarization observations have revealed a hidden BLR (e.g. Tran et al. 1992; Tran 1995; Shu et al. 2007). The nuclear continuum polarization is $\lesssim 1.5 \%$, significantly lower than that of the broad lines, implying that the majority of the optical continuum cannot be due to scattered light from the hidden type 1 nucleus. The blue central source is instead associated with a compact dusty starburst (effective radius $<0.2 \mathrm{kpc}$ ) which occurred $\sim 6 \mathrm{Myr}$ ago in a brief period of time and whose light dominates from the ultraviolet to the near-infrared (NIR; Heckman et al. 1997, González Delgado et al.1998). This is therefore a starburst-AGN hybrid system. MRK 477 has a starforming rate $\mathrm{SFR}=24 \mathrm{M}_{\odot} \mathrm{yr}^{-1}$ inferred from the infrared (IR) luminosity (assuming that it is starburst-dominated) $\log \frac{L_{\mathrm{IR}}}{L_{\odot}}=11.14$ (Krug et al.2010). This places it in the regime of luminous IR galaxies (LIRGs: $11 \leqslant \log \frac{L_{\mathrm{IR}}}{L_{\odot}}<12$ ). The detection of the Wolf Rayet (WR) blue bump around the He II $\lambda 4686$ line by Heckman et al. (1997) led these authors to propose that MRK 477 is a luminous member of the class of WR galaxies.

The QSO2 host harbors a supermassive black hole with a mass in the range $\log \left(M_{\mathrm{BH}}\right) \sim 7.18$ (as derived from the $M_{\mathrm{BH}}$ versus $\sigma_{*}$ correlation) to 8.84 (as inferred from the polarimetric $\mathrm{H} \beta$ line; Zhang, Bian \& Huang 2008).

Although radio quiet according to the $L_{\text {[OIII) }}$ versus $L_{\text {rad }}$, radio loud versus radio quiet, classification criteria (explained in Villar-Martín et al.2014), MRK 477 shows a clear excess of radio emission compared to that expected from the stellar contribution. The $8.4 \mathrm{GHz}$ Very Large Array (VLA) radio continuum map ( 0.26 arcsec resolution) shows a triple radio source $(\sim 1.2$ arcsec total extension) whose morphology correlates with that of the narrow line region (NLR). The size and overall northeast-southwest axis of the radio structures are shared by the [OIII] emission, which extends up to a similar distance to the north-east (Heckman et al. 1997).

Shuder \& Osterbrock (1981) reported for the first time that the emission lines have a large blue-ward asymmetry (i.e., excess) at low intensity level relative to the continuum. Villar Martín et al. (2014) proposed that the ionized nuclear outflow responsible for such an asymmetry has been triggered by the interaction between the radio source and the NLR. These authors proposed that negative feedback can be triggered by the radio structures in a significant fraction of radio quiet quasars. Thanks to its high intrinsic luminosity

1 The HST images were retrieved from the HST Science Legacy Archive. They are part of the programmes 9379 with principal investigator PI H. Schmitt (ACS/HRC image), 8597 with PI M. Michael Regan (WFPC image) and 7330 with PI J. Mulchaey (NICMOS image). and closeness, MRK 477 is an excellent test object to gain further insight into this mechanism.

We present here a detailed study of the optical and near-infrared spectra of MRK 477. We explore a diversity of aspects that provide a more complete understanding of the nature of this object, example of a type 2 quasar in the nearby universe, as well as a starburst-AGN hybrid system. The paper is organized as follows. The spectra are described in Sect. 2, together with the spectral fitting procedure. We present results in Sect. 3 regarding line identification, correlations between the gas kinematics and a diversity of parameters (ionization potential, critical density), the spatial extension of the ionized gas, the ionized outflow, the presence of narrow $\mathrm{Fe}^{+}$emission lines, the coronal line spectrum and the detection of WR features. The results are discussed in Sect. 4 and the conclusions are presented in Sect. 5 .

We assume $\Omega_{\Lambda}=0.7, \Omega_{\mathrm{M}}=0.3, H_{0}=71 \mathrm{~km} \mathrm{~s}^{-1} \mathrm{Mpc}^{-1}$. At $z=0.037,1$ arcsec corresponds $0.725 \mathrm{kpc}$ (Wright 2006).

\section{DATA SET}

\subsection{Optical spectrum}

The optical spectrum was obtained as part of the Sloan Digital Sky Survey (SDSS, York et al. 2000). It spans the rest frame range $\sim 3660-8880 \AA$ range (Figs. 2 and 3 ). It corresponds to an aperture defined by the 3 arcsec diameter SDSS fibre $(\sim 2.2 \mathrm{kpc}$ at $z=0.037)$ centred at the galaxy nucleus. For comparison, the triple radio source identified by Heckman et al. (1997) has a total extension of $\sim 1.2$ arcsec and thus it is well within the fibre area.

The spectral resolution is $\sim 180 \pm 20 \mathrm{~km} \mathrm{~s}^{-1}$. All values of emission line full width at half-maximum (FWHM) have been corrected for instrumental broadening.

The main emission lines have large equivalent widths and the stellar features are comparatively weak. Underlying stellar absorption of the Balmer lines is expected to be negligible and therefore subtracting the stellar continuum is not necessary for our purposes.

\subsection{NIR spectrum: observations and data reduction}

NIR $\mathrm{H}+\mathrm{K}$ long-slit spectra were obtained with the NIR camera/spectrometer LIRIS (Long-slit Intermediate Resolution Infrared Spectrograph; Acosta-Pulido et al. 2003; Manchado et al. 2004), attached to the Cassegrain focus of the 4.2 m William Herschel Telescope (WHT). LIRIS is equipped with a Rockwell Hawaii $1024 \times 1024 \mathrm{HgCdTe}$ array detector, whose spatial scale is 0.25 arcsec pixel ${ }^{-1}$.

The NIR observations were performed as part of the Isaac Newton Group service programme (programme SW2014b22) in two different nights, 28 March 2015 and 02 May 2015. The H+K grism was used. It covers the 1.388$2.419 \mu \mathrm{m}$ spectral range and provides a dispersion of $9.7 \AA$ $\mathrm{pix}^{-1}$. The seeing size during the March observations was FWHM $\sim 2^{\prime \prime}$ (as measured from the standard star). A $1^{\prime \prime}$ wide slit was used oriented at position angle $\mathrm{PA}=43^{\circ}$ East of North. This PA is aligned with the radio axis and the NLR axis as seeing in the HST image presented by Heckman et al. (1997). The resulting spectral resolution was $35.7 \pm 2.2 \AA$ (or 


\section{Mrk 477}

WFPC2 F606W

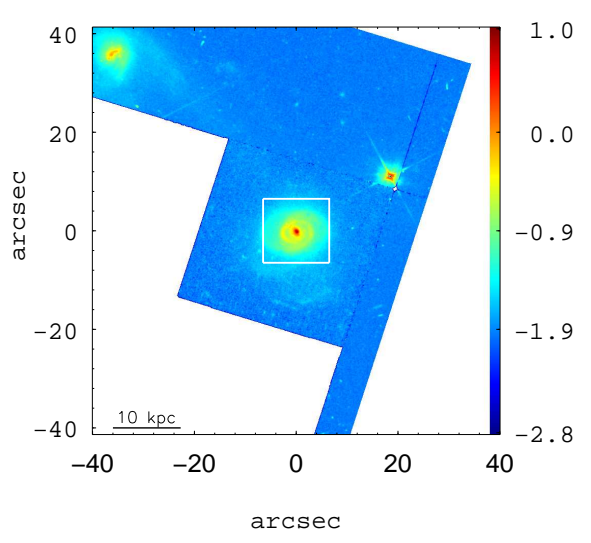

ACS F330W

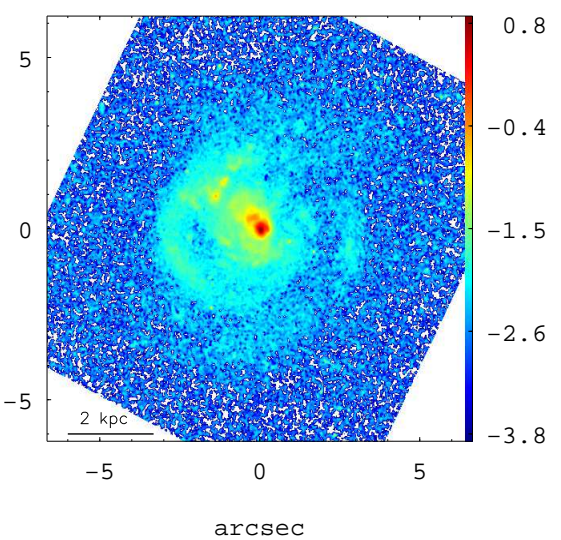

NICMOS F160W

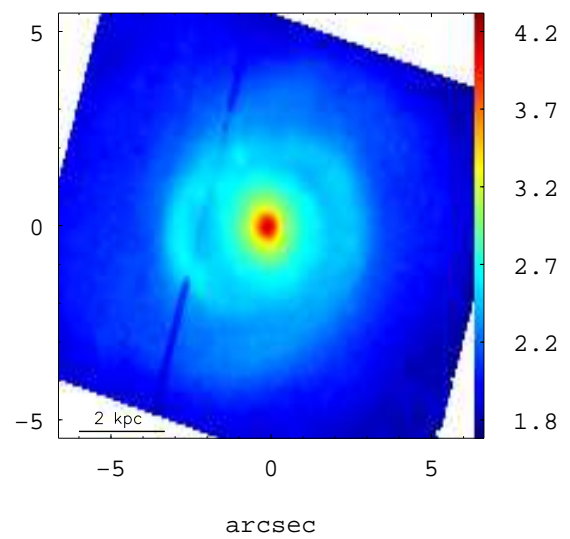

Figure 1. Hubble Space Telescope (HST) images of MRK 477. They were obtained with Wide-Field Planetary Camera 2 (WFPC2, F606W filter, left), the Advanced Camera for Surveys (ACS/HRC, F330W filter, middle) and the near-infrared Camera and Multi-Object Spectrometer (NICMOS, F160W filter, right) respectively. The flux intensity maps are represented in logarithmic scale such that F [erg $\left.\mathrm{s}^{-2} \mathrm{~cm}^{-2} \AA^{-1} \operatorname{arcsec}^{-2}\right]=10^{A+b}$, where $A$ is a constant factor (i.e., $-18.3,-14.5$ and -16.4 , respectively, for WFPC2, ACS and NICMOS images) and $b$ is indicated in the coloured bar. The white box in the WFPC2 image identifies the FoV covered by the ACS and NICMOS images. The horizontal line at the bottom left of these images corresponds to a scale of $10 \mathrm{kpc}$ for the WFPC2 image and to a scale of 2 kpc for the ACS and NICMOS images. The spatial scale for this galaxy is $0.725 \mathrm{kpc} / \operatorname{arcsec}$. North is at the top and East to the left in all the panels.

$550 \pm 34 \mathrm{~km} \mathrm{~s}^{-1}$ at the observed wavelength of $\left.\mathrm{Pa} \alpha\right)$. In Fig. 4 we show the NIR spectrum extracted from a $3.5^{\prime \prime}$ aperture, centred at the position of the nucleus. The total exposure time on source was $6400 \mathrm{~s}(16 \times 400 \mathrm{~s})$.

The May data were obtained with seeing $\mathrm{FWHM}=0.65 \pm 0.05^{\prime \prime}$ (measured from the standard star). A $0.75^{\prime \prime}$ slit was used, also with $\mathrm{PA}=43^{\circ}$. The resulting spectral resolution was $23.2 \pm 1.2 \AA$ (or $318 \pm 18 \mathrm{~km} \mathrm{~s}^{-1}$ ). The observations were performed through clouds and the signal to noise ratio of the final spectrum was low. In spite of this, the good seeing conditions allowed to obtain more accurate information on the spatial extension of the strongest emission line $\mathrm{Pa} \alpha$. The total exposure time on source was $4000 \mathrm{~s}(10 \times 400 \mathrm{~s})$. All FWHM values of the NIR spectra have been corrected for instrumental broadening.

The data were reduced following standard procedures for near-IR spectroscopy, using the LIRISDR dedicated software within the IRAF 2 enviroment. For a detailed description of the reduction process see Ramos Almeida et al. (2009). Consecutive pairs of AB two-dimensional spectra were subtracted to remove the sky background. The resulting frames were then wavelength calibrated and flat-fielded before registering and co-adding all frames to provide the final spectra.

The absolute flux calibration of the March spectrum is intended to be an approximation since the spectra of the comparison star are likely subjected to slit losses due to centring and tracking errors. We note that in this work we refer to flux ratios only, and therefore the uncertainty in the

2 IRAF is distributed by the National Optical Astronomy Observatory, which is operated by the Association of Universities for the Research in Astronomy, Inc., under cooperative agreement with the National Science Foundation (http://iraf.noao.edu/). absolute flux calibration of the spectra does not affect our results.

\subsection{Spectral fitting}

In order to study the kinematic and physical properties of the nuclear ionized outflow we have fitted the spectral profiles of numerous emission lines (Sect. 3). For this we used the STARLINK package DIPSO. This software is based on the optimization of fit coefficients, in the sense of minimizing the sum of the squares of the deviations of the fit from the spectrum data. The output from a completed fit consists of the optimized parameters (Gaussian central $\lambda$, FWHM, intensity peak, flux) and their errors (calculated in the linear approximation, from the error matrix).

Two methods were attempted. In method I, we assumed that all emission lines have the same kinematic substructure as [OIII] $\lambda \lambda 4959,5007$ (same number of kinematic components with identical FWHM in $\mathrm{km} \mathrm{s}^{-1}$ and relative velocities, as explained in Villar Martín et al. 2014). In physical terms, this method corresponds to a situation where all gaseous regions emit all lines, although with different relative fluxes.

On the other hand, this is not necessarily the case and some lines might come from different regions, possibly resulting in variations of the kinematic substructure from line to line. As an example, low critical density lines $\left(\sim\right.$ few $\times 10^{3}$ $\left.\mathrm{cm}^{-3}\right)$ are quenched in high density gas with $n>10^{6} \mathrm{~cm}^{-3}$. To account for this possibility, whenever possible we have also attempted to fit the lines without applying prior kinematic restrictions (method II).

Also, all fits that produced unphysical results were rejected even if mathematically valid (e.g. a fit producing [NII] $\frac{\lambda 6583}{\lambda 6548}$ very different from the theoretical value 3.0 ).

As we will see the lines are complex with multiple kine- 
matic components and they are often severely blended with neighbour lines so that the fits are sometimes complicated and not unambiguous. Applying methods I and II whenever possible we account for the uncertainties involved in a more realistic way than using a single method.

Both methods do not always produce acceptable results. For instance, the $[\mathrm{SII}] \lambda \lambda 4068,4076+\mathrm{H} \delta$ and $[\mathrm{SII}] \lambda \lambda 6716,6731$ blends could not be successfully fitted applying full constraints from the [OIII] lines. The opposite occurs with the $\mathrm{H} \alpha+[\mathrm{NII}]$ blend.

Methods I and II could be successfully applied to $\mathrm{H} \gamma$, $[\mathrm{OIII}] \lambda 4363, \mathrm{H} \beta$ and $[\mathrm{OI}] \lambda 6300$. It is found that, $[\mathrm{OIII}] \lambda 4363$ is the most uncertain. While the narrow and intermediate components isolated in the fits (see below) are consistent within 30 and 10 per cent, respectively, with the two methods, a discrepancy of a factor of $\sim 60$ per cent is found for the broadest component. The results for all three kinematic components isolated in the fits of the other three lines differ by $<20$ per cent. These uncertainties will be taken into account when relevant.

\section{RESULTS}

\subsection{Line identification.}

We identify $\sim 90$ emission lines in the SDSS optical spectrum of MRK 477 (Table 11), many of them detected for the first time in this object. For some features the identification is ambiguous and/or it could be the contribution of several emission lines. In such cases, all the possible identifications or contributors are quoted separated with ";". As an example, a broad feature with an asymmetric profile is detected at $\lambda \sim 5272 \AA$. This is identified as "[Fe III];[FeII];[FeII];[Fe VII]" because it might be the blend of [FeIII] $\lambda 5270.4$, [FeII] $\lambda \lambda 5273.4,5276.0$ and [FeVII] $\lambda 5276.4$. When two or more lines are known to contribute to a given feature, all are quoted and separated with "+". For instance, the $[\mathrm{OII}]$ at $\lambda \sim 3727$ and $[\mathrm{NI}]$ at $\lambda \sim 5200$ doublets are shown as " $3727.0+3728.8$ " and "5197.9+5200.4".

The works by Verón-Cetty, Joly \& Véron (2004) and Verón-Cetty et al. (2013) have been used for iron (Fe) line identifications. The critical densities $n_{\text {crit }}$ of forbidden transitions are quoted in column 5 of Table 1 when available. Most have been computed with the PYNEB package optimized for the analysis of emission lines (Luridiana, Morisset \& Shaw 2014). These have been estimated for $T_{e} \sim 15,000 \mathrm{~K}$. The rest have been retrieved from De Robertis \& Osterbrock (1984).

The lines identified in the NIR spectrum are shown in Table 2

\subsection{Relationships between the kinematics and} $n_{\text {crit }}, \mathbf{I P}_{\text {low }}$ and $\mathbf{I P}$ high .

We plot in Fig. 5 the FWHM (top panels) and the velocity shift $V_{S}$ (bottom) versus the critical density ( $n_{\text {crit }}$, Table 1), the lower $\left(\mathrm{IP}_{\text {low }}\right)$ and upper ( $\left.\mathrm{IP}_{\text {high }}\right)$ ionization potentials for a subsample of $\sim 28$ optical and 2 NIR forbidden emission

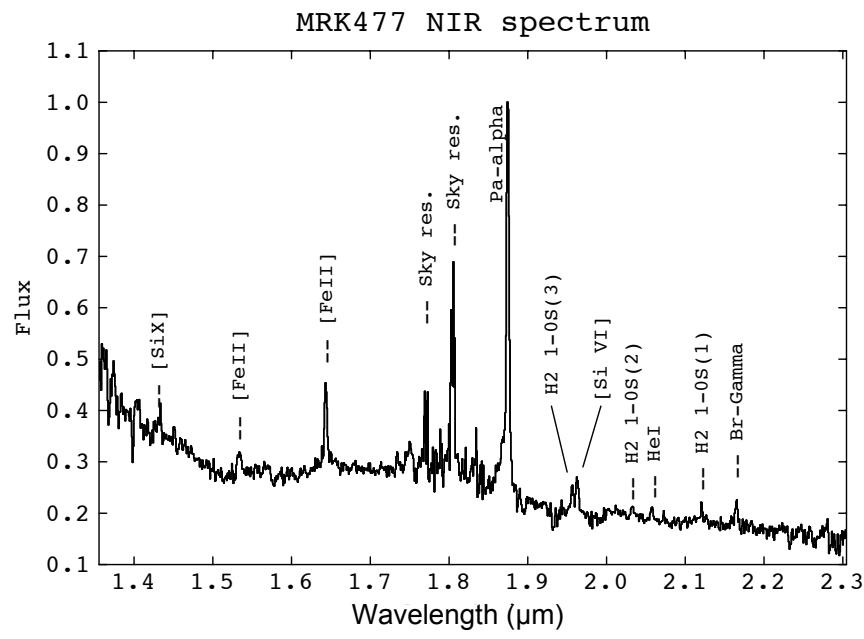

Figure 4. NIR LIRIS-WHT spectrum of MRK 477 extracted from a $\sim 1^{\prime \prime} \times 3.5^{\prime \prime}$ aperture centered on the nucleus. The detected lines are indicated. Flux is in units of $\times 10^{-15} \mathrm{erg} \mathrm{s}^{-1} \mathrm{~cm}^{-2} \mathrm{~A}^{-1}$ and $\lambda$ in $\mu \mathrm{m}$.

lines ${ }^{3} V_{S}$ corresponds to the difference between $\lambda_{\text {air }}$ and $\lambda_{\text {obs }}$ in Table 1. where $\lambda_{\text {air }}$ is the vacuum wavelength and $\lambda_{\text {obs }}$ is that measured from the spectrum corrected for $z$. Only lines for which both parameters could be measured with reasonable accuracy are plotted. As an example, this was not the case for $[\mathrm{SiX}] \lambda 1.430$ and $[\mathrm{FeII}] \lambda 1.257$ due to the weakness of the lines and/or the distortion of the line profiles by sky residuals. The optical and NIR lines span a wide range in critical densities $\left(\sim 1.3 \times 10^{3}-6.3 \times 10^{8} \mathrm{~cm}^{-3}\right)$ and ionization potentials ( $\left.\mathrm{IP}_{\text {high }} \sim 13-205 \mathrm{eV}\right)$. A significant correlation is found between the FWHM and the critical density (Spearman correlation coefficients $r_{s}=0.79$ and $p=0.000001$, excluding [FeII] $\lambda 1.644)$, as already found by De Robertis \& Osterbrock (1986) whose data are shown as open circles. No significant correlation is found with the ionization potentials $\left(r_{s}=0.43\right.$ and $p=0.02$ for FWHM versus $\mathrm{IP}_{\text {low }}$ and $r_{s}=0.42$ and $p=0.03$ for FWHM versus $\left.\mathrm{IP}_{\text {high }}\right)$. No trends are found either for $V_{S}$ except maybe an apparent preference for redshifts $\left(V_{S}>0\right)$ for lines with the highest critical densities.

The NIR [FeII] $\lambda 1.644$ line is a clear outlier in Fig. 5. being too broad compared to what is expected for its critical density $\left(5.6 \times 10^{4} \mathrm{~cm}^{-3}\right)$ and ionization potentials (7.9 and $16.2 \mathrm{eV}$ ) according to the general trends defined by the other lines. We will discuss this in more detail below.

The correlation between FWHM and $n_{\text {crit }}$ has been found in many type 1 and type 2 AGNs and it holds for AGN luminosities that differ by a factor of up to $\sim 5000$ (Espey 1994). $V_{S}$, on the other hand, shows a different behavior from object to object (e.g. Appenzeller \& Östreicher 1988).

The FWHM versus $n_{\text {crit }}$ correlation implies that the line emission from the NLR originates in different subregions under different physical conditions and kinematic properties with a broad density range. Higher critical density lines (many of which have also very high ionization potentials) are broader because they are emitted predominantly by higher

3 The FWHM and $V_{S}$ values have been derived from single Gaussian fits to the emission lines. 


\begin{tabular}{|c|c|c|c|c|}
\hline $\begin{array}{l}(1) \\
\text { Species }\end{array}$ & $\begin{array}{l}(2) \\
\lambda_{\text {air }} \\
(\AA)\end{array}$ & $\begin{array}{l}(3) \\
\lambda_{\text {obs }} \\
(\AA)\end{array}$ & $\frac{{ }^{(4)}}{F \operatorname{lu} x(\mathrm{H} \beta)}$ & $\begin{array}{l}(5) \\
n_{c r i t} \\
\left(\mathrm{~cm}^{-3}\right)\end{array}$ \\
\hline $\begin{array}{l}{[\mathrm{OII}]} \\
\mathrm{H} 12\end{array}$ & $\begin{array}{l}3727.0+3728.8 \\
3750.2\end{array}$ & $\begin{array}{l}3726.9 \\
3750.1\end{array}$ & $\begin{array}{l}2.26 \pm 0.18 \\
0.014 \pm 0.003\end{array}$ & $(1.3 / 4.5) \times 10^{3}$ \\
\hline$[\mathrm{FeVII}]$ & $\begin{array}{l}3750.2 \\
3758.9\end{array}$ & $\begin{array}{l}3750.1 \\
3759.3\end{array}$ & $\begin{array}{l}0.014 \pm 0.003 \\
0.046 \pm 0.005\end{array}$ & $3.7 \times 10^{7 a}$ \\
\hline H11 & 3770.6 & 3770.4 & $0.023 \pm 0.002$ & \\
\hline H10 & 3797.9 & 3797.5 & $0.026 \pm 0.003$ & \\
\hline H9 & 3835.4 & 3835.5 & $0.045 \pm 0.003$ & \\
\hline$[\mathrm{NeIII}]$ & 3868.8 & 3868.6 & $1.04 \pm 0.03$ & $1.3 \times 10^{7}$ \\
\hline $\mathrm{HeI}+\mathrm{H} 8$ & $3888.7+3889.05$ & 3888.8 & $0.16 \pm 0.02$ & \\
\hline $\begin{array}{l}{[\mathrm{NeIII}]+\mathrm{H} 7} \\
\mathrm{HeI}\end{array}$ & $\begin{array}{l}3967.5+3970.1 \\
4026.2\end{array}$ & 3968.2 & $0.43 \pm 0.04$ & $1.3 \times 10^{7}$ \\
\hline $\begin{array}{l}\text { HeI } \\
\text { [SII] }\end{array}$ & $\begin{array}{l}4026.2 \\
4068.6\end{array}$ & $\begin{array}{l}4026.1 \\
4068.6\end{array}$ & $\begin{array}{l}0.013 \pm 0.002 \\
0.19 \pm 0.01\end{array}$ & $3.0 \times 10^{6}$ \\
\hline [SII] & 4076.4 & 4076.1 & $0.065 \pm 0.03$ & $1.5 \times 10^{6}$ \\
\hline $\mathrm{H} \delta$ & 4101.7 & 4101.5 & $0.24 \pm 0.02$ & \\
\hline$[\mathrm{FeV}]$ & 4180.9 & 4180.7 & $0.008 \pm 0.002$ & \\
\hline FeII $] ;[\mathrm{FeV}]$ & $4227.2 ; 4228.0$ & $4228.2 \pm 0.1$ & $0.017 \pm 0.002$ & \\
\hline$[\mathrm{FeII}]$ & 4244.0 & 4244.5 & $0.019 \pm 0.002$ & \\
\hline$[\mathrm{FeII}]$ & 4276.8 & $4278.3 \pm 0.1$ & $0.013 \pm 0.002$ & \\
\hline$[\mathrm{FeII}]$ & 4287.4 & $4289.1 \pm 0.4$ & $0.014 \pm 0.002$ & \\
\hline $\mathrm{H} \gamma$ & 4340.5 & 4340.31 & $0.45 \pm 0.05$ & \\
\hline [OIII] & 4363.2 & 4363.2 & $0.23 \pm 0.02$ & $3.4 \times 10^{7}$ \\
\hline$[\mathrm{FeII}]$ & 4416.3 & 4416.4 & $0.021 \pm 0.002$ & \\
\hline HeI & 4471.4 & 4472.2 & $0.035 \pm 0.002$ & \\
\hline$[\mathrm{FeII}]$ & 4570.0 & 4570.9 & $0.018 \pm 0.005$ & \\
\hline$[\mathrm{FeIII}] ; \mathrm{CIV}$ & 4658.1 & 4658.7 & $0.034 \pm 0.001$ & \\
\hline HeII & 4685.7 & 4685.7 & $0.138 \pm 0.008$ & \\
\hline$[\mathrm{FeIII}]$ & 4701.5 & 4700.2 & $0.010 \pm 0.008$ & \\
\hline$[$ ArIV] & 4711.4 & 4711.5 & $0.011 \pm 0.002$ & $1.7 \times 10^{4}$ \\
\hline$[\mathrm{NeIV}]$ & $4725.5 ; 4726.8$ & 4725.1 & $0.008 \pm 0.003$ & $(6.2 / 2.5) \times 10^{7}$ \\
\hline [ArIV] & 4740.2 & 4740.2 & $0.016 \pm 0.003$ & $1.6 \times 10^{5}$ \\
\hline [FeIII] & 4754.7 & $4754.0 \pm 0.5$ & $0.007 \pm 0.002$ & \\
\hline$[\mathrm{FeIII}]$ & 4769.5 & 4769.6 & $0.006 \pm 0.002$ & \\
\hline [FeIII] & 4777.7 & 4777.5 & $0.007 \pm 0.002$ & \\
\hline$[\mathrm{Fe}$ II $]$ & 4814.5 & $4816.3 \pm$ & $0.016 \pm 0.002$ & \\
\hline $\mathrm{H} \beta$ & 4861.3 & 4861.3 & 1.00 & \\
\hline [FeVII] & 4893.4 & 4893.9 & $0.008 \pm 0.002$ & \\
\hline [OIII] & 4958.9 & 4958.9 & $3.7 \pm 0.3$ & $8.6 \times 10^{5}$ \\
\hline [OIII] & 5006.8 & 5006.8 & $10.5 \pm 0.3$ & $8.6 \times 10^{5}$ \\
\hline$[\mathrm{FeVI}]$ & 5145.8 & 5145.9 & $0.007 \pm 0.002$ & $2.7 \times 10^{7 a}$ \\
\hline$[\mathrm{FeVII}]$ & 5158.9 & 5159.4 & $0.042 \pm 0.005$ & $3.3 \times 10^{6} a$ \\
\hline$[\mathrm{FeVI}]$ & 5176.4 & 5177.0 & $0.010 \pm 0.001$ & $3.3 \times 10^{7 a}$ \\
\hline$[\mathrm{NI}]$ & $5197.9+5200.4$ & 5199.2 & $0.05 \pm 0.01$ & $(1.6 / 0.51) \times 10^{3}$ \\
\hline$[\mathrm{Fe} \mathrm{III}] ;[\mathrm{FeII}] ;[\mathrm{FeII}] ;[\mathrm{Fe} \mathrm{VII}]$ & $5270.4 ; 5273.4 ; 5276.0 ; 5276.4$ & 5272.1 & $0.058 \pm 0.004$ & \\
\hline$[\mathrm{CaV}]$ & 5309.1 & 5309.7 & $0.032 \pm 0.002$ & $5.1 \times 10^{7}$ \\
\hline$[\mathrm{FeVI}]$ & 5335.2 & 5335.5 & $0.017 \pm 0.002$ & \\
\hline$[\mathrm{FeII}]$ & 5379.0 & 5379.7 & $0.011 \pm 0.002$ & \\
\hline $\mathrm{HeII} ;[\mathrm{FeII}]$ & $5411.5 ; 5412.7$ & 5412.0 & $0.015 \pm 0.005$ & \\
\hline $\mathrm{FeII}] ;[\mathrm{FeVI}]$ & $5425.3 ; 5425.7$ & 5424.1 & $0.018 \pm 0.006$ & \\
\hline$[\mathrm{FeVI}]$ & 5485.0 & 5484.5 & $0.005 \pm 0.001$ & \\
\hline$\left[\begin{array}{lll}\mathrm{Cl} & \mathrm{III}\end{array}\right]$ & 5517.7 & 5517.5 & $0.006 \pm 0.002$ & $8.5 \times 10^{3}$ \\
\hline$[\mathrm{ArX}] ;[\mathrm{FeII}] ; \mathrm{FeII}]$ & $5533.0 ; 5527.6 ; 5534.8$ & 5531.39 & $0.013 \pm 0.003$ & \\
\hline$\left[\begin{array}{ll}\mathrm{Cl} & \mathrm{III}\end{array}\right]$ & 5537.9 & 5537.9 & $0.009 \pm 0.002$ & $2.9 \times 10^{4}$ \\
\hline$[\mathrm{OI}]$ & 5577.34 & 5577.9 & $0.014 \pm 0.001$ & $9.0 \times 10^{7}$ \\
\hline$[\mathrm{FeVI}]$ & 5631.1 & 5630.9 & $0.008 \pm 0.002$ & \\
\hline$[\mathrm{FeVI}]$ & 5677.0 & 5677.1 & $0.005 \pm 0.001$ & \\
\hline$[\mathrm{FeVII}]$ & 5720.9 & $5721.8 \pm 0.2$ & $0.047 \pm 0.002$ & $3.6 \times 10^{7 a} a$ \\
\hline$[\mathrm{NII}]$ & 5754.6 & 5754.7 & $0.040 \pm 0.002$ & $1.8 \times 10^{7}$ \\
\hline $\mathrm{HeI}$ & 5875.6 & 5875.7 & $0.131 \pm 0.007$ & \\
\hline$[\mathrm{FeVII}]$ & 6086.9 & 6087.7 & $0.12 \pm 0.01$ & $3.6 \times 10^{7 a}$ \\
\hline$[\mathrm{OI}]$ & 6300.3 & 6300.5 & $0.81 \pm 0.02$ & $1.1 \times 10^{6}$ \\
\hline [SIII] & 6312.1 & 6311.8 & $0.016 \pm 0.003$ & $1.6 \times 10^{7}$ \\
\hline$[\mathrm{OI}]$ & 6363.8 & 6365.0 & $0.26 \pm 0.02$ & $1.1 \times 10^{6}$ \\
\hline$[\mathrm{ArV}]$ & 6435.1 & 6435.7 & $0.010 \pm 0.002$ & $1.4 \times 10^{7}$ \\
\hline$[\mathrm{NII}]$ & 6548.1 & 6548.1 & $0.53 \pm 0.05$ & $1.2 \times 10^{5}$ \\
\hline $\mathrm{H} \alpha$ & 6562.8 & 6562.8 & $3.9 \pm 0.2$ & \\
\hline$[\mathrm{NII}]$ & 6583.5 & 6583.4 & $1.5 \pm 0.1$ & $1.2 \times 10^{5}$ \\
\hline $\mathrm{HeI}$ & 6678.2 & 6678.7 & $0.035 \pm 0.005$ & \\
\hline [SII] & 6716.4 & 6716.5 & $0.60 \pm 0.03$ & $1.5 \times 10^{3}$ \\
\hline [SII] & 6730.8 & 6730.9 & $0.75 \pm 0.03$ & $4.0 \times 10^{3}$ \\
\hline$[\mathrm{ArV}]$ & 7005.8 & 7005.7 & $0.014 \pm 0.003$ & $1.3 \times 10^{7}$ \\
\hline $\mathrm{HeI}$ & 7065.7 & 7065.3 & $0.07 \pm 0.005$ & \\
\hline [ArIII $]$ & 7135.8 & 7135.8 & $0.20 \pm 0.01$ & $6.4 \times 10^{6}$ \\
\hline$[\mathrm{FeII}]$ & 7155.2 & 7156.8 & $0.06 \pm 0.01$ & \\
\hline$[\mathrm{FeII}]$ & 7172.0 & 7173.8 & $0.014 \pm 0.004$ & \\
\hline$[\mathrm{CaII}]$ & 7291.5 & 7292.8 & $0.02 \pm 0.01$ & \\
\hline [OII] & 7318.9 & 7321.0 & $0.23 \pm 0.04$ & $6.5 \times 10^{6}$ \\
\hline$[\mathrm{OII}]$ & 7339.7 & 7329.2 & $0.32 \pm 0.04$ & $6.5 \times 10^{6}$ \\
\hline$[\mathrm{NiII}]$ & 7377.8 & 7379.1 & $0.040 \pm 0.008$ & \\
\hline$[\mathrm{FeII}]$ & 7452.6 & $7453.9 \pm 0.5$ & $0.016 \pm 0.002$ & \\
\hline $\mathrm{FeI} ;[\mathrm{FeII}]$ & $7634.0 ; 7637.5$ & $7639 \pm 2$ & $0.007 \pm 0.002$ & \\
\hline$[$ ArIII $]$ & 7751.1 & 7750.9 & $0.062 \pm 0.003$ & $6.4 \times 10^{6}$ \\
\hline MgII; [NiIII]; [FeXI]; MgII & $7877.0 ; 7889.9 ; 7891.8 ; 7896.0$ & 7888.8 & $0.023 \pm 0.003$ & \\
\hline MgII & 8232.0 & $8232.7 \pm 0.5$ & $0.020 \pm 0.005$ & \\
\hline $\mathrm{OI}$ & 8446.0 & 8446.6 & $0.029 \pm 0.002$ & \\
\hline $\mathrm{HI} \mathrm{Pa} 14$ & 8598.5 & 8595.4 & $0.010 \pm 0.003$ & \\
\hline$[\mathrm{FeII}]$ & 8605.5 & 8604.5 & $0.033 \pm 0.004$ & \\
\hline$[\mathrm{FeII}]$ & 8617.0 & 8617.8 & $0.05 \pm 0.02$ & \\
\hline $\mathrm{HI} \mathrm{Pa} 12$ & 8750.6 & 8750.2 & $0.013 \pm 0.002$ & \\
\hline
\end{tabular}

Table 1. Emission lines identified in the SDSS spectrum of MRK 477. The observed line wavelengths $\lambda^{\text {obs }}$ (column 3) have been determined by fitting a single Gaussian to the emission lines. Errors are quoted when they provide relevant information regarding possible multiple identifications or there is a significant shift between the observed and the air wavelengths (column 2). The line ratios (column 4) are given relative to $\mathrm{H} \beta$, with $F(\mathrm{H} \beta)=(1.01 \pm 0.04) \times 10^{-13} \mathrm{erg} \mathrm{s}^{-1} \mathrm{~cm}^{-2}$. They are not corrected for reddening. The critical densities are quoted when available. Superscript ${ }^{a}$ in column (5) indicates that $n_{\text {crit }}$ has been taken from De Robertis \& Osterbrock (1984). The other $n_{\text {crit }}$ values have been computed with the PYNEB software (see the text). 


\begin{tabular}{|c|c|c|c|}
\hline Comp. & $\begin{array}{l}\text { FWHM } \\
\left(\mathrm{km} \mathrm{s}^{-1}\right)\end{array}$ & $\begin{array}{l}V_{S} \\
\left(\mathrm{~km} \mathrm{~s}^{-1}\right)\end{array}$ & $\frac{F_{i}}{F(\mathrm{H} \beta)}$ \\
\hline & & \multicolumn{2}{|l|}{$[\mathrm{SII}] \lambda 4068$} \\
\hline Narrow & $94 \pm 37$ & $-2 \pm 8$ & $0.07 \pm 0.01$ \\
\hline Interm. & $505 \pm 35$ & $17 \pm 10$ & $0.26 \pm 0.02$ \\
\hline Broad & $1534 \pm 97$ & $-376 \pm 85$ & $0.25 \pm 0.05$ \\
\hline & & \multicolumn{2}{|l|}{$[\mathrm{SII}] \lambda 4076$} \\
\hline Narrow & $94 \pm 37$ & $-2 \pm 8$ & $0.010 \pm 0.006$ \\
\hline Interm. & $505 \pm 35$ & $17 \pm 10$ & $0.12 \pm 0.02$ \\
\hline \multirow[t]{2}{*}{ Broad } & $1534 \pm 97$ & $-376 \pm 85$ & $0.08 \pm 0.07$ \\
\hline & & \multicolumn{2}{|l|}{$\mathrm{H} \delta$} \\
\hline Narrow & $94 \pm 37$ & $-2 \pm 8$ & $0.23 \pm 0.04$ \\
\hline Interm. & $505 \pm 35$ & $17 \pm 10$ & $0.23 \pm 0.06$ \\
\hline \multirow[t]{2}{*}{ Broad } & $1534 \pm 97$ & $-376 \pm 85$ & $0.26 \pm 0.03$ \\
\hline & & \multicolumn{2}{|l|}{$\mathrm{H} \gamma$} \\
\hline Narrow & $157 \pm 28$ & $-7 \pm 7$ & $0.47 \pm 0.03$ \\
\hline Interm. & $529 \pm 40$ & $36 \pm 10$ & $0.38 \pm 0.04$ \\
\hline Broad & $1830 \pm 133$ & $-224 \pm 51$ & $0.57 \pm 0.06$ \\
\hline & & \multicolumn{2}{|l|}{$[\mathrm{OIII}] \lambda 4363$} \\
\hline Narrow & $157 \pm 28$ & $-7 \pm 7$ & $0.11 \pm 0.02$ \\
\hline Interm. & $529 \pm 40$ & $36 \pm 10$ & $0.43 \pm 0.03$ \\
\hline Broad & $1830 \pm 133$ & $-224 \pm 51$ & $0.27 \pm 0.08$ \\
\hline & & \multicolumn{2}{|l|}{$\mathrm{H} \beta$} \\
\hline Narrow & $137 \pm 22$ & $-2 \pm 6$ & $(4.1 \pm 0.4) \times 10^{-14}$ \\
\hline Interm. & $471 \pm 22$ & $7 \pm 7$ & $(3.7 \pm 0.1) \times 10^{-14}$ \\
\hline Broad & $1527 \pm 75$ & $-188 \pm 24$ & $(2.3 \times 0.1) \times 10^{-14}$ \\
\hline & & \multicolumn{2}{|l|}{$[\mathrm{OIII}] \lambda 5007$} \\
\hline Narrow & $177 \pm 15$ & $5 \pm 9$ & $8.5 \pm 0.9$ \\
\hline Interm. & $545 \pm 15$ & $22 \pm 11$ & $13.1 \pm 0.5$ \\
\hline Broad & $1839 \pm 53$ & $-227 \pm 23$ & $9.6 \pm 0.7$ \\
\hline & & \multicolumn{2}{|l|}{$[\mathrm{OI}] \lambda 6300$} \\
\hline Narrow & $211 \pm 11$ & $-4 \pm 5$ & $0.60 \pm 0.07$ \\
\hline Interm. & $563 \pm 29$ & $17 \pm 6$ & $0.91 \pm 0.08$ \\
\hline Broad & $1745 \pm 40$ & $-257 \pm 24$ & $1.08 \pm 0.07$ \\
\hline & & \multicolumn{2}{|l|}{$\mathrm{H} \alpha$} \\
\hline Narrow & As [OIII] & As [OIII] & $3.1 \pm 0.3$ \\
\hline Interm. & As [OIII] & As [OIII] & $4.9 \pm 0.2$ \\
\hline Broad & As [OIII] & As [OIII] & $4.2 \pm 0.3$ \\
\hline & & \multicolumn{2}{|l|}{$[\mathrm{NII}] \lambda 6583$} \\
\hline Narrow & As [OIII] & As [OIII] & $1.0 \pm 0.1$ \\
\hline Interm. & As [OIII] & As [OIII] & $1.70 \pm 0.07$ \\
\hline Broad & As [OIII] & As [OIII $]$ & $0.9 \pm 0.2$ \\
\hline & & \multicolumn{2}{|l|}{$[\mathrm{SII}] \lambda 6716$} \\
\hline Broad & $186 \pm 9$ & $-3 \pm 5$ & $0.67 \pm 0.07$ \\
\hline Interm. & $501 \pm 17$ & $-4 \pm 6$ & $0.68 \pm 0.04$ \\
\hline Broad & $1397 \pm 76$ & $-493 \pm 89$ & $0.30 \pm 0.08$ \\
\hline & & \multicolumn{2}{|l|}{$[\mathrm{SII}] \lambda 6731$} \\
\hline Narrow & $186 \pm 9$ & $-3 \pm 5$ & $0.66 \pm 0.07$ \\
\hline Interm. & $501 \pm 17$ & $-4 \pm 6$ & $0.80 \pm 0.03$ \\
\hline Broad & $1397 \pm 76$ & $-493 \pm 89$ & $0.77 \pm 0.08$ \\
\hline
\end{tabular}

Table 3. Results of the spectral decomposition of the main lines in the spectrum of MRK 477. Three kinematic components are isolated in all cases, including a broad blueshifted component emitted by the ionized outflow. No values are quoted for $\mathrm{H} \alpha$ and [NII] because successful fits could only be obtained with full constraints from the [OIII] lines (see Sect. 2.1). The flux of each component relative to $\mathrm{H} \beta$ is given in the last column. The exception is $\mathrm{H} \beta$, for which the actual fluxes are quoted.

velocity, high density and high ionization regions closer to the central engine.

We have done $n$ estimations using the densitydiagnostic line ratios $[\mathrm{SII}] \lambda 6716 / \lambda 6731,[\mathrm{Cl} \mathrm{III}] \lambda 5517 / \lambda 5537$ and $[$ ArIV $] \lambda 4711 / \lambda 4740$, which are not affected by extinction or $T_{e}$ variations. They are sensitive to different $n$ regimes. The [SII] ratio $\left(n_{\text {crit }}=1.5 \times 10^{3}\right.$ and $4.0 \times 10^{3}$ $\mathrm{cm}^{-3}$, respectively) is sensitive to $n$ variations $\sim$ several $\times 10^{3}$ $\mathrm{cm}^{-3}$. These lines are suppressed in high density re-

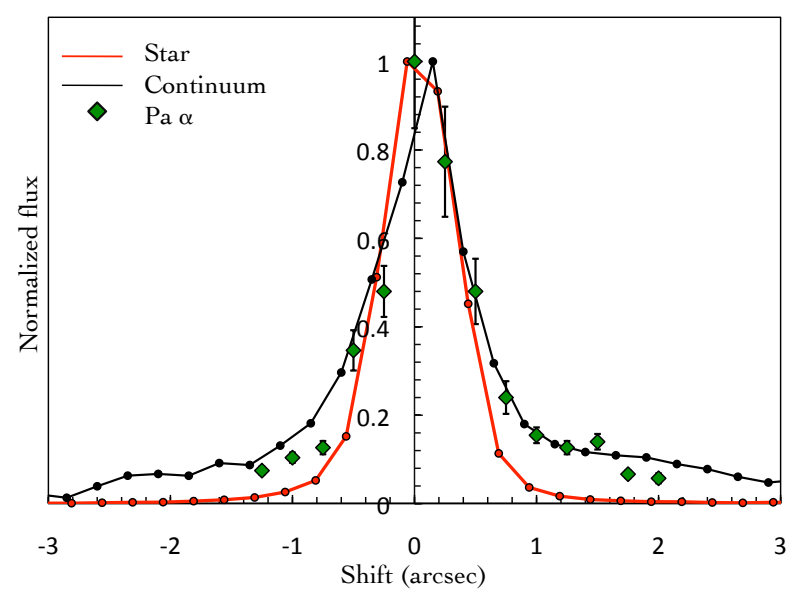

Figure 6. Spatial profiles (normalized fluxes) of the NIR continuum and Pa $\alpha$ along PA $43^{\circ} \mathrm{N}$ to E, compared with the seeing disk (star). Both the continuum and $\mathrm{Pa} \alpha$ are spatially extended.

gions by collisional de-excitation. Other line pairs that can be overcome this limitation are [Cl III] $\lambda 5517 / \lambda 5537$ $\left(n_{\text {crit }}=8.5 \times 10^{3}\right.$ and $\left.2.9 \times 10^{4} \mathrm{~cm}^{-3}\right)$, sensitive at $n \sim 10^{4}$ $\mathrm{cm}^{-3}$ and $[\mathrm{ArIV}] \lambda 4711 / \lambda 4740\left(n_{\text {crit }}=1.7 \times 10^{4}\right.$ and $1.6 \times 10^{5}$ $\mathrm{cm}^{-3}$ ) at $n \sim 10^{4}-10^{5} \mathrm{~cm}^{-3}$.

For $\quad$ MRK $477, \quad[\mathrm{SII}] \lambda 6716 / \lambda 6731=0.70 \pm 0.06$, $[\mathrm{ClIII}] \lambda 5517 / \lambda 5537=0.74 \pm 0.22$ and $[\mathrm{ArIV}] \lambda 4711 / \lambda 4740$ $=0.67 \pm 0.09$, impling densities $n=1945_{-460}^{+670} \mathrm{~cm}^{-3}$ (from [SII]), $7250_{-5100}^{+13,300}$ (from [Cl III]) and $n=14,700_{-3750}^{+6150}$ (from [ArIV]). So, the [SII] doublet implies the existence of gas with $n \sim 2000 \mathrm{~cm}^{-3}$, the [Cl III] doublet, in spite of the larger uncertainties suggests higher densities and the [ArIV] doublet confirms the existence of gas with $n>10^{4} \mathrm{~cm}^{-3}$ in the NLR of MRK 477.

\subsection{Spatial extension of the ionized gas}

The only available information about the spatial distribution of the ionized gas in MRK 477 is provided by the [OIII] image presented by Heckman et al. (1997). It shows a bright knot or ridge of emission at $\sim 0.4 \operatorname{arcsec}$ to the northeast of the central source and aligned with the radio axis. This knot is not resolved from the nucleus in our NIR spectrum, for which the slit was roughly aligned along the same axis. We find, on the other hand, that $\mathrm{Pa} \alpha$ is extended at both sides of the central source. We show in Fig. [6 the spatial profiles of (1) a star observed during the May run (seeing size FWHM $=0.65 \pm 0.05),(2)$ the MRK 477 continuum and (3) the $\mathrm{P} \alpha$ flux, with the underlying continuum subtracted. Although more compact than the continuum, $\mathrm{Pa} \alpha$ is extended, as demonstrated by the clear excess above the seeing wings, up to a maximum radial distance of $\sim 2$ arcsec or $\sim 1.5 \mathrm{kpc}$ to the NE.

\subsection{The ionized outflow}

Villar Martín et al. (2014) performed a kinematic and ionization characterization of the nuclear ionized outflow in MRK 477. Its signature is a broad blueshifted kinematic component in the $[\mathrm{OIII}] \lambda \lambda 4959,5007$ lines, with 

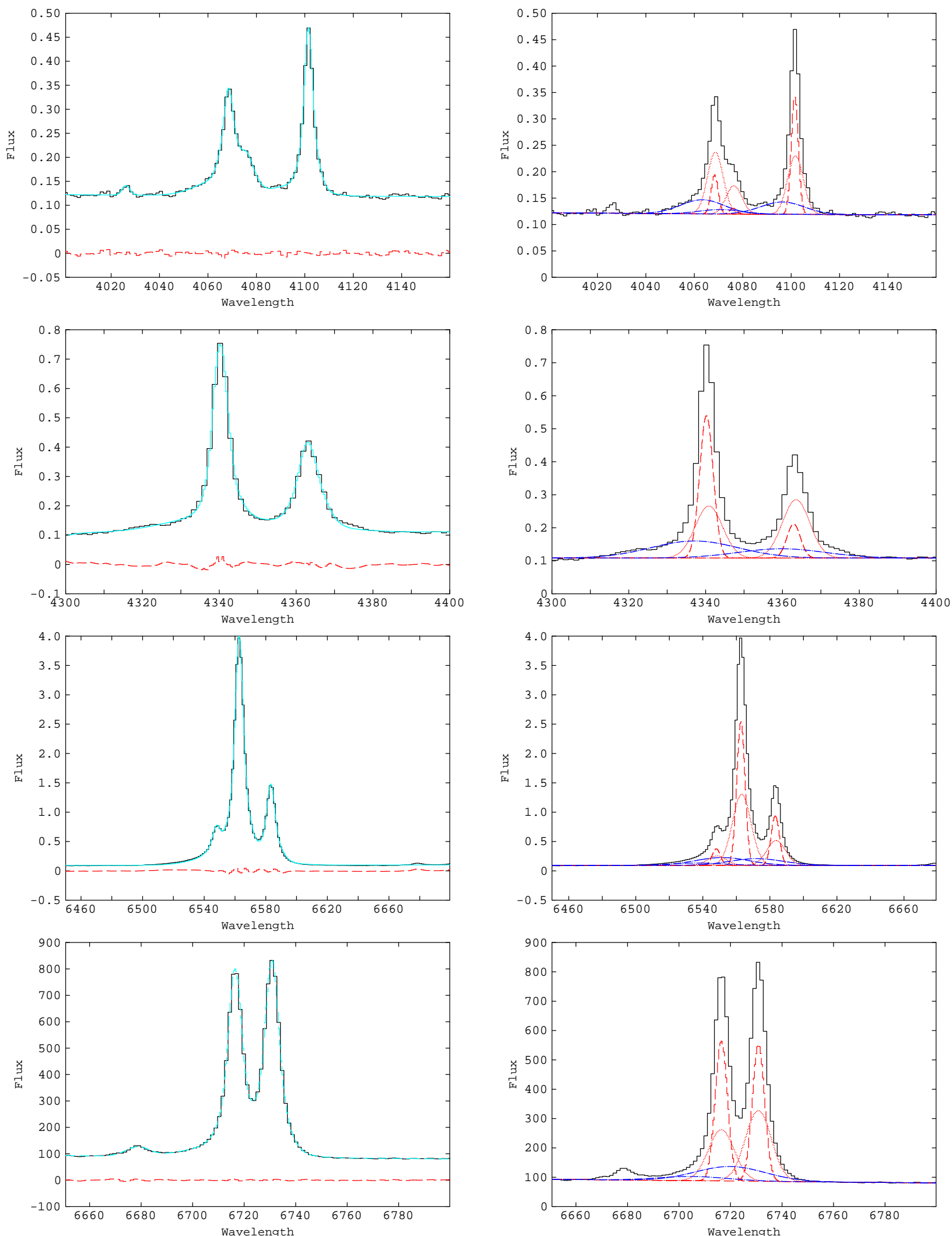

Figure 7. Left: data (black), fits (cyan) and residuals (dashed-red) for a diversity of emission lines. Right: individual kinematic components isolated in each line. Different line styles are used for different kinematic components. The same red colour is used for components with the same redshift: long-dashed red (narrow component), dotted-red (intermediate component), dot-dashed blue (broad component; i.e. the outflow emission). From top to bottom: [SII] $\lambda \lambda 4068,4076$ and $\mathrm{H} \delta ; \mathrm{H} \gamma$ and [OIII] $\lambda 4363 ;[\mathrm{NII}] \lambda \lambda 6548,6583$ and $\mathrm{H} \alpha$; [SII] $\lambda \lambda 6716,6731$. 

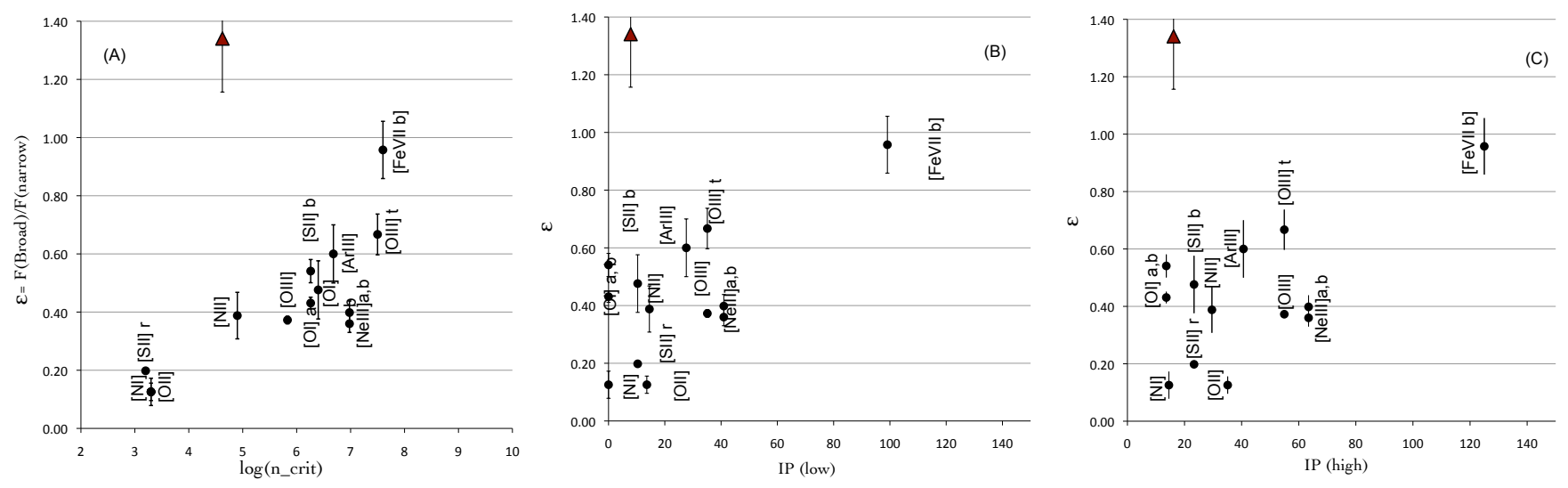

Figure 8. $\epsilon=\frac{F_{\text {broad }}}{F_{\text {narrow }}}$ parametrizes the relative contribution between the outflow flux emission and the rest of the line flux. The correlation with the critical density (panel A) implies that the outflow emission is relatively stronger in higher density gas. [OI] 'a' and ' $\mathrm{b}$ ' correspond to the $\lambda 6300$ and $\lambda 6312$ lines respectively; [NeIII]' $a$ ' and ' $b$ ' to $\lambda 3869$ and $\lambda 3967$; [SII] 'b' and 'r' refer to the blue and red [SII] doublets at $\lambda \lambda 6048,6076$ and $\lambda \lambda 6716,6731$ respectively. [FeII] $\lambda 1.644 \mu \mathrm{m}$ (red triangle) is again an outlier.

FWHM $1850 \mathrm{~km} \mathrm{~s}^{-1}$ and $V_{S} \sim-225 \mathrm{~km} \mathrm{~s}^{-1}$. Based on the high turbulence of this gaseous component, the line ratios consistent with AGN (rather than stellar) excitation processes and the relatively high contribution of the outflowing gas to the total line fluxes, the authors proposed that it has been triggered by the 1.2 arcsec scale radio source. This is supported by the correlation between the radio and [OIII] morphologies (Heckman et al. 1997), which demonstrates that the radio source is interacting with the NLR.

\subsubsection{Isolating the outflow emission in a diversity of emission lines}

By means of the kinematic decomposition of the spectral profiles, we have isolated the emission from the outflowing gas in numerous emission lines (Sect. 2.1). Our goal is to characterize its physical properties and to constrain more accurately its spatial location.

We show in Table 3 the results of the fits for several optical emission lines (see also Fig. 7). FWHM and $V_{S}$ correspond to those fits where no prior constraints from [OIII] were applied (method II, see Sect 2.3). When both methods could be applied, the flux values and errors account for the dispersion allowed by them.

Generally, we find rather coherent results for all strong lines for which multiple component fitting procedures could be applied. By "coherent" it is meant that all lines consist of three kinematic components (Table [3): two of them have similar $z$ and are relatively narrow $(\mathrm{FWHM} \sim[95,210]$ and $[470,560] \mathrm{km} \mathrm{s}^{-1}$ respectively). A third broad blueshifted component is moreover isolated in all lines, which is emitted by the outflowing gas. It has FWHM $[1400,1840 \mathrm{~km}$ $\mathrm{s}^{-1}$ and $V_{S} \sim[-490,-190] \mathrm{km} \mathrm{s}^{-1}$. All the three kinematic components have line ratios consistent with type 2 AGN, as already pointed out by Villar Martín et al. (2014).

Other lines show clear evidence of a broad underlying component, although the triple Gaussian fit is not possible due to the low signal-to-noise and/or the complex blend with neighbour lines. We have calculated the ratio $\epsilon=\frac{F_{\text {broad }}}{F_{\text {narrow }}}$ between the flux of the broad (outflowing) component and the rest of the line flux (which for simplicity we will name $F_{\text {narrow}}$, although it contains the narrow and intermediate components) for as many lines as possible. This gives a measurement of the relative contribution between the most turbulent outflowing gas and the more quiescent ambient gas.

The ratio $\epsilon$ is plotted against $\log \left(n_{\text {crit }}\right), \mathrm{FWHM}, \mathrm{IP}_{\text {low }}$ and $\mathrm{IP}_{\text {high }}$ in Fig. 8. A significant correlation is found with the critical density $\left(r_{s}=0.73\right.$ and $p=0.003$; panel A). No significant trend is found with the ionization potentials $\left(r_{s}=0.40\right.$ and $p=0.15$ for $\epsilon$ versus $\mathrm{IP}_{\text {low }}$ and $r_{s}=0.35$ and $p=0.23$ for $\epsilon$ versus $\left.\mathrm{IP}_{\text {high }}\right)$.

An interesting case is the high ionization line $[\mathrm{FeVII}] \lambda 6087$, for which a very broad component is isolated with FWHM $=2460 \pm 340 \mathrm{~km} \mathrm{~s}^{-1}$ (Fig. 9) and $V_{S}=-150 \pm 60$ $\mathrm{km} \mathrm{s}^{-1}$. Thus, the line which shows simultaneously very high critical density and ionization potential, also shows the most extreme kinematics and the largest $\epsilon=0.96 \pm 0.10$ of all optical lines. At the other end, the lowest $n_{\text {crit }}$ (also low ionization) lines [NI] $\lambda 5200$ and $[\mathrm{SII}] \lambda \lambda 6716,6731$, are the narrowest $\left(\mathrm{FWHM} \sim 300 \mathrm{~km} \mathrm{~s}^{-1}\right.$ ) and have the smallest $\epsilon \sim 0.1-0.2$ (Fig. 8).

The correlation between $\epsilon$ and the FWHM suggests that the increasing broadening of the lines is due to the increasing outflow influence. The correlation with $n_{\text {crit }}$ shows that the outflow emission is relatively stronger in higher density gas. Ultimately, these results suggest that the FWHM versus $n_{\text {crit }}$ correlation is produced by the outflow in MRK 477.

[FeII $] \lambda 1.644$ is the only forbidden NIR line for which the spectral decomposition could be applied. It consists of a narrow, spectrally unresolved component with $\mathrm{FWHM} \lesssim 340$ $\mathrm{km} \mathrm{s}^{-1}$ and a very broad and prominent underling component with $\mathrm{FWHM}=4770 \pm 830 \mathrm{~km} \mathrm{~s}^{-1}$ (Fig. 10) shifted by $315 \pm 231 \mathrm{~km} \mathrm{~s}^{-1}$ relative to the narrow core. It is the only line for which the broad component is not blueshifted. For comparison, $\mathrm{Pa} \alpha$ consists of two components with FWHM $\lesssim 260 \mathrm{~km} \mathrm{~s}^{-1}$ and $2240 \pm 230 \mathrm{~km} \mathrm{~s}^{-1}$ respectively 

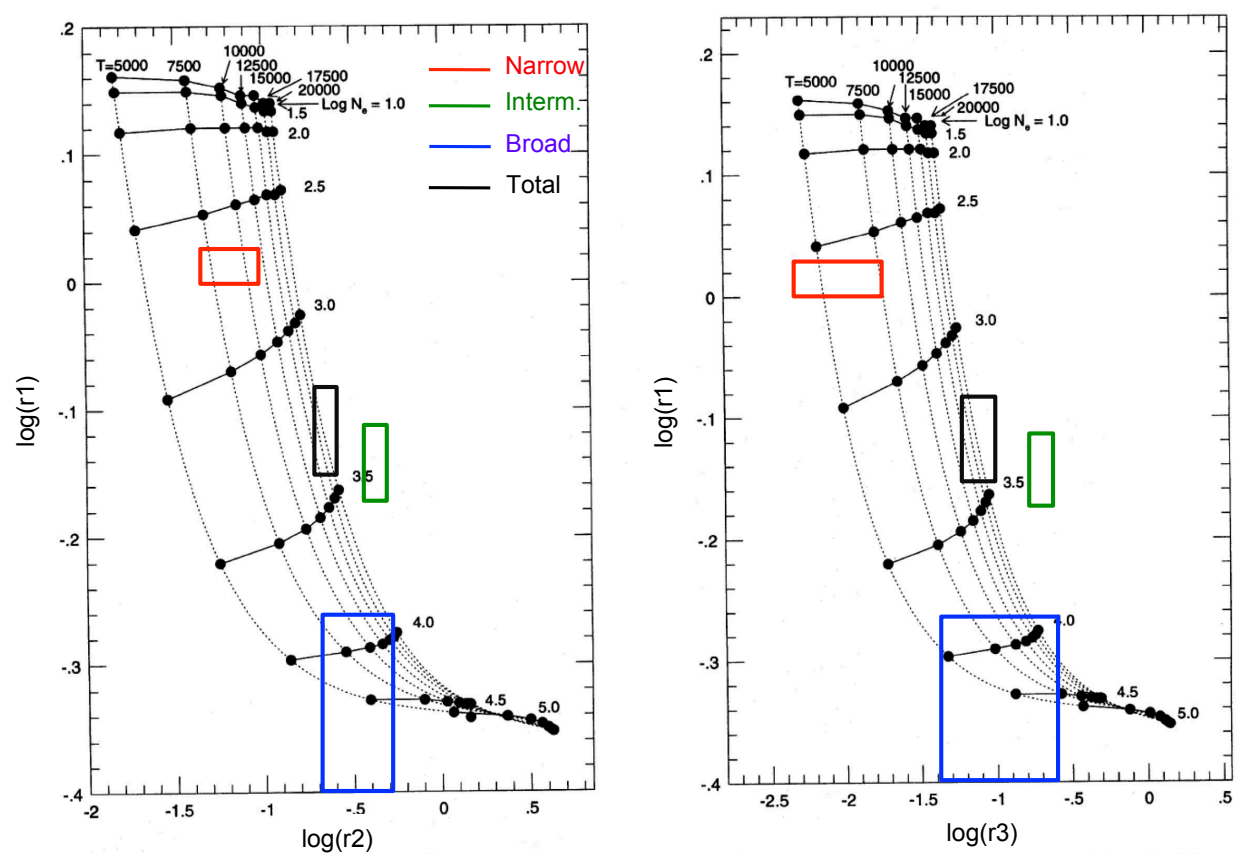

Figure 11. Constraints on the electron density $n$ for the three kinematic components and for the integrated lines (adapted from Fig. 2 and 3 in Keenan et al. 1996)). A trend is found such that the broader the lines, the higher the density. Thus, the outflowing gas has the highest density.

\begin{tabular}{llllllllll}
\hline$(1)$ & $(2)$ & $(3)$ & $(4)$ & $(5)$ & $(6)$ & $(7)$ & $(8)$ & $(9)$ & $(10)$ \\
Comp. & $\frac{\mathrm{H} \alpha}{\mathrm{H} \beta}$ & $c_{\mathrm{H} \alpha}$ & $\mathrm{E}(\mathrm{B}-\mathrm{V})_{\mathrm{H} \alpha}$ & $\frac{\mathrm{H} \gamma}{\mathrm{H} \beta}$ & $c_{\mathrm{H} \gamma}$ & $\mathrm{E}(\mathrm{B}-\mathrm{V})_{\mathrm{H} \gamma}$ & $\frac{\mathrm{H} \delta}{\mathrm{H} \beta}$ & $c_{\mathrm{H} \delta}$ & $\mathrm{E}(\mathrm{B}-\mathrm{V})_{\mathrm{H} \delta}$ \\
\hline Narrow & $3.1 \pm 0.3$ & $0.14 \pm 0.12$ & $0.08 \pm 0.08$ & $0.47 \pm 0.03$ & $0.09 \pm 0.09$ & $0.04 \pm 0.03$ & $0.23 \pm 0.04$ & $0.37 \pm 0.37$ & $0.24 \pm 0.11$ \\
Intermediate & $4.9 \pm 0.2$ & $0.70 \pm 0.04$ & $0.47 \pm 0.03$ & $0.38 \pm 0.06$ & $0.62 \pm 0.46$ & $0.42 \pm 0.19$ & $0.23 \pm 0.06$ & $0.40 \pm 0.40$ & $0.26 \pm 0.12$ \\
Broad & $4.2 \pm 0.3$ & $0.49 \pm 0.08$ & $0.32 \pm 0.06$ & $0.57 \pm 0.09$ & 0.00 & 0.00 & $0.26 \pm 0.03$ & $0.15 \pm 0.15$ & $0.09 \pm 0.05$ \\
\hline Total & $4.0 \pm 0.2$ & $0.45 \pm 0.06$ & $0.29 \pm 0.05$ & $0.45 \pm 0.05$ & $0.21 \pm 0.21$ & $0.13 \pm 0.06$ & $0.24 \pm 0.02$ & $0.27 \pm 0.23$ & $0.17 \pm 0.08$ \\
\hline
\end{tabular}

Table 4. Extinction correction $(c$ and $\mathrm{E}(\mathrm{B}-\mathrm{V}))$ determined from the Balmer ratios (columns 2,5 and 8 ) for the three kinematic components and the integrated lines.

\begin{tabular}{lllllll}
\hline$(1)$ & $(2)$ & $(3)$ & $(4)$ & $(5)$ & $(6)$ & $(7)$ \\
Comp. & $r 1^{\text {obs }}=r 1^{\text {int }}$ & $r 2^{\text {obs }}$ & $r 2^{\text {int }}$ & $r 3^{\text {obs }}$ & $r 3^{\text {int }}$ & $n\left(\mathrm{~cm}^{-3}\right)$ \\
\hline Narrow & $1.03 \pm 0.03$ & $0.05 \pm 0.01$ & $0.07 \pm 0.02$ & $0.007 \pm 0.005$ & $0.010 \pm 0.007$ & $\sim 400-630$ \\
Intermediate & $0.73 \pm 0.05$ & $0.17 \pm 0.02$ & $0.37 \pm 0.09$ & $0.08 \pm 0.01$ & $0.17 \pm 0.04$ & $\sim 2000-4000$ \\
Broad & $0.41 \pm 0.12$ & $0.24 \pm 0.05$ & $0.34 \pm 0.14$ & $0.08 \pm 0.07$ & $0.25 \pm 0.11$ & $\gtrsim 8000$ \\
\hline Total & $0.76 \pm 0.06$ & $0.13 \pm 0.01$ & $0.22 \pm 0.03$ & $0.05 \pm 0.01$ & $0.08 \pm 0.02$ & $\sim 1600-3200$ \\
\hline
\end{tabular}

Table 5. Density sensitive line ratios observed $\left({ }^{\text {obs }}\right)$ and corrected $\left({ }^{\text {int }}\right)$ for reddening. $r 1=\frac{[\mathrm{SII}] \lambda 6716}{[\mathrm{SII}] \lambda 6731}, r 2=\frac{[\mathrm{SII}] \lambda 4068}{[\mathrm{SII}] 6716+6731}, r 3=$ $\frac{[\mathrm{SII}] 4076}{[\mathrm{SII}] 6716+6731}$. The range of densities $n$ has been calculated using $r 1$. A trend is found such that the broader the component, the higher
the density.

(ii) Electron density $n$. We have constrained $n$ with the ratio $r 1=\frac{[\mathrm{SII}] \lambda 6716}{\operatorname{SII} \lambda \lambda 6731}$ (Osterbrock 1989). The results are shown in Table [5] The apparently non perturbed ambient gas (the narrow component) has $n \sim 400-630 \mathrm{~cm}^{-3}$. The intermediate component, which shows intermediate kinematic and physical properties, has $n \sim 2,000-4,000 \mathrm{~cm}^{-3}$. Finally, the most extreme kinematic component, which is associated with the outflowing gas has the highest density $n \gtrsim 8.000$ $\mathrm{cm}^{-3}$.

We have further checked these results using $r 2=$
$\frac{[\mathrm{SII}] 4068}{\operatorname{SII} 6716+6731}$ and $r 3=\frac{[\mathrm{SII}] 4076}{[\mathrm{SII}] 6716+6731}$ (Keenan et al. 1996). [SII] $6716+6731$
Unlike $r 1$, these ratios have a strong dependence on the electron temperature $T_{e}$ and reddening. They are therefore less efficient at constraining $n$, but they provide a useful test. The dependence of both ratios with $n$ and $T_{e}$ can be seen in Fig. 11.

To correct for reddening (eq. [2]), for each component we adopt $c$ as the average of the maximum and minimum values obtained from the three Balmer ratios (Table 4). The uncertainties are calculated using half the difference between 
2 AGN (Penston et al. 1984, Rose et al. 2013) cannot be confirmed either.

The highest optical ionization lines unambiguosly identified are $[\mathrm{NeV}] \lambda \lambda 3346,3426\left(\mathrm{IP}_{\text {low }}=97 \mathrm{eV}\right.$, out of the SDSS spectral range; reported by De Robertis 1987) and the [FeVII] lines $\left(\mathrm{IP}_{\text {low }}=99 \mathrm{eV}\right)$ at $\lambda 5159,6087 \AA$ (also reported by De Robertis 1987), and lines at 3759, 4893, $5721 \AA$. Also possibly [FeVII] at $5276 \AA$, [ArX] at $5533 \AA$, although other identifications cannot be discarded (Table 1).

With somewhat lower ionization level, [CaV] at 5309 $\AA \quad\left(\mathrm{IP}_{\text {low }}=67 \mathrm{eV}\right)$ and several $[\mathrm{FeVI}]\left(\mathrm{IP}_{\text {low }}=75 \mathrm{eV}\right)$ lines at $5146,5176,5335,5485,5631,5677 \AA$ are also detected and possibly $[\mathrm{FeV}] \lambda 5726\left(\mathrm{IP}_{\text {low }}=55 \mathrm{eV}\right)$ and $[\mathrm{FeVI}]$ at $5426 \AA$.

$[\mathrm{FeVII}] \lambda 6086$ is fainter relative to low ionization lines (e.g. $[\mathrm{OI}] \lambda 6300$ ) than usually found in active galaxies with strong coronal emission. $\frac{[\mathrm{FeVII}] \lambda 6086}{[\mathrm{OI}] \lambda 6300}=0.15 \pm 0.01$ for MRK 477. For comparison, the Seyfert 1 and 2 galaxies studied by Rodríguez Ardila et al. (2006) with detected $[\mathrm{FeX}]$ and $[\mathrm{FeXI}]$ lines show in general (4 out of 5 objects) $\frac{[\mathrm{FeVII}] \lambda 6086}{[\mathrm{OI}] \lambda 6300}$ in the range $\sim 0.6-5.7$.

\subsection{The WR bumps}

Heckman et al. (1997) detected a broad emission complex around He II $\lambda 4686$, which is clearly appreciated in the SDSS spectrum. They fitted this so called "blue bump" satisfactorily as a blend of the He II line together with other lines which are identified in Fig. 15](top). This unresolved bump is a blend of lines emitted mainly by late WN and early WC WR stars, although some contribution of early WN stars might be present (Schaerer \& Vacca 1998). Heckman et al. (1997) propose that it may be produced by an ensemble of about 30,000 WR stars (WN subtype), in which case MRK 477 would be a luminous (but not extraordinarily so) member of the class of WR galaxies.

Another feature often identified in WR galaxies due to WR stars is the so called "red bump" due to broad CIV $\lambda 5808$ emission, emitted mainly by WCE stars. This is usually much weaker than the "blue bump" and was not detected by Heckman et al. (1997)

We show in Fig. 15 (middle panel) the MRK 477 spectrum near the "red bump" and the optical spectrum of the WR galaxy SBS 1222+614 (Guseva, Izotov \& Thuan 2000) for comparison (bottom panel). The appearance of the "red bump" in this and other WR galaxies is very similar to that in MRK 477. We thus believe that the "red bump" is detected in the SDSS spectrum of this QSO2. Its very large width (FWHM $\sim 80 \AA$ or $\sim 4100 \mathrm{~km} \mathrm{~s}^{-1}$ ) rules out as its origin the broad wings due to the ionized outflow identified in many other emission lines.

\section{DISCUSSION}

We have analysed the optical and NIR spectra of MRK 477, the nearest obscured quasar. WR features first identified by Heckman et al. (1997) and confirmed here by the new detection of the "red bump" at $\sim 5800 \AA$ (Sect. 3.7) show that it has undergone very recent star formation.

The optical+NIR spectrum of MRK 477 is very rich, with $\sim 100$ detected emission lines ( $\sim 90$ in the optical). In spite of the lack of spatial information of the SDSS spectrum, the spectral decomposition of numerous lines has allowed us to characterize, at least partially, the spatial structure and the gradients in the physical and kinematic properties of the gas.

\subsection{The origin of the FWHM versus $n_{\text {crit }}$ correlation}

As in many other type 1 and type 2 AGN, a significant correlation has been found between the FWHM of the lines and the critical density $n_{\text {crit }}$ (in log). Based on the analysis of eight type 1 AGN spectra, Stern, Laor \& Baskin (2014; S14 hereafter) showed that this relation is consistent with the velocity field within the black hole gravitational sphere of influence (radius $R_{g}$ ), assuming the $n \propto R^{-2}$ relation implied by the equilibrium between the NLR clouds and the radiation pressure and that the emission of each forbidden line is dominated by gas with $n \sim n_{\text {crit }}$. This explanation implies that beyond some threshold $n_{\text {crit }}, n_{\text {crit }}^{0}$, which depends on the luminosity in Eddington units $\dot{m}$, the line emission will be dominated by gas within $R_{g}$, and therefore is expected to show larger velocities than gas which kinematics is dominated by the host galaxy. For MRK 477, the stellar velocity dispersion $\sigma_{*}=117 \mathrm{~km} \mathrm{~s}^{-1}$ (Zhang, Bian \& Wang 2008), $L_{\mathrm{bol}}$ and the values of $M_{\mathrm{BH}}$ quoted in Sect. 1 imply $R_{g} \sim 1$ and 40 for $\log \left(M_{\mathrm{BH}}\right)=7.19$ and 8.84 respectively (eq. 26 in S14). This implies a threshold $n_{\text {crit }}^{0}=10^{8.6}$ $\mathrm{cm}^{-3}$ (eq. 6 in $\left.\mathrm{S} 14\right)$ for $\log \left(M_{\mathrm{BH}}\right)=7.19$. This value is much larger than the $n_{\text {crit }} \sim 10^{4.5}-10^{5.5} \mathrm{~cm}^{-3}$ where the FWHM starts to increase in MRK 477 (top-left panel in Fig. 5), which makes the gravitational interpretation unlikely. For $\log \left(M_{\mathrm{BH}}\right)=8.84, n_{\text {crit }}^{0}=10^{5.4} \mathrm{~cm}^{-3}$ is indeed consistent with the data. The success of this prediction supports the gravitational interpretation for the FWHM versus $n_{\text {crit }}$ correlation, provided MRK 477 harbors such a massive black hole. On the other hand, the kinematic substructure of the lines raises doubts about whether the emission is truly emitted mostly by gas at $n \sim n_{\text {crit. }}$. As an example, the [OIII] line flux is dominated by gas with $n \lesssim 4000 \mathrm{~cm}^{-3}$ (see Sect. 3.4).

Alternatively, we propose that the outflow produces this correlation in MRK 477. Thisis supported by the correlations between $\epsilon=\frac{F_{\text {broad }}}{F_{\text {narrow }}}$ and $n_{\text {crit }}$ (Sect. 3.3.1), which imply that the outflow is relatively stronger and is responsible for the increasing line broadening at increasing densities. This correlation does not differ in any statistical sense from the usual FWHM versus $n_{\text {crit }}$ one. The difference lies in the physical interpretation we propose.

It is possible that the effects of ionized outflows explain the FWHM versus $n_{\text {crit }}$ correlation in other AGN.

Three kinematic components have been isolated in the main optical emission lines. The narrowest and intermediate components, have similar $z\left(V_{S} \sim 0 \mathrm{~km} \mathrm{~s}^{-1}\right)$ and are relatively narrow (FWHM $[95-210]$ and $[470-560]$ $\mathrm{km} \mathrm{s}^{-1}$ respectively). A third broad blueshifted component is moreover isolated in all lines with FWHM in the range $\sim[1400-1840] \mathrm{km} \mathrm{s}^{-1}$ and $V_{S} \sim[-490-(-190)] \mathrm{km} \mathrm{s}^{-1}$. The narrow component is likely to trace the NLR ambient, non perturbed gas, while the broad component is emitted by the most turbulent, outflowing gas. The intermediate component traces gas of intermediate properties. All three components have line ratios consistent with type 2 active galaxies, as already pointed out by Villar Martín et al. (2014), 
implying that they are spatially located within the quasar ionization cones.

The difference in kinematics is associated with a difference in physical properties. The broader the component, the higher the density. The sequence we find is $n \sim(400-630)$ $\mathrm{cm}^{-3}$ for the ambient gas, $n \sim(2000-4000) \mathrm{cm}^{-3}$ for the intermediate component and $n \gtrsim 8000 \mathrm{~cm}^{-3}$ for the outflowing gas (see §3.3.2). Higher $n$ of the outflowing gas is also suggested by the correlation between $\epsilon=\frac{F_{\text {broad }}}{F_{\text {narrow }}}$ and $n_{\text {crit }}$ for different forbidden lines (see above). Density enhancement of the outflowing gas has been found in some radio galaxies and QSO2 (e.g. Holt et al. 2011; Villar Martín et al. 2014).

\subsection{The spatial location of nuclear ionized outflow}

The isolation of the broad component in numerous emission lines demonstrates that the outflow involves gas covering a large range of ionization potentials and critical densities (at least $\mathrm{IP}_{\text {high }} \sim 8$ to $125 \mathrm{eV}, \log \left(n_{\text {crit }}\right) \sim 3.3$ to 7.5$)$. We try to constrain next the spatial location where it was triggered and how far its effects extend.

Bennert et al. (2006a,b) found that the observed gas density of the NLR gas decreases with increasing distance to the AGN in Seyfert 1 and 2 galaxies (see also S14). A similar behavior is expected in MRK 477. The difference in kinematic and physical properties between the broad, intermediate and narrow components can be naturally explained if they are located at increasing distance from the AGN, with the outflow emission dominating at smaller distances, in the inner part of the NLR or even closer (see also Villar-Martín et al. 2014). The outflow becomes weaker as it propagates outwards and reaches less dense regions, where lines of lower critical densities are preferentially emitted. Because the outflow has lost some power, it drags less and less mass and its emission becomes weaker relative to the ambient gas, which dominates the line fluxes in the most distant, lower density regions. This can also explain the correlation of $\epsilon$ with $n_{\text {crit }}$.

If the radiation pressure is responsible for the NLR density gradient (S14), the estimated $n$ for the three kinematic components will help to constrain the distance from the central engine $R$ at which each one is preferentially emitted. The expected behaviour of $n$ with the ionizing luminosity $L_{i o n}$ and $R$ is predicted to be ( $\underline{\mathrm{S} 14})$ :

$$
n=7 \times 10^{4} L_{\text {ion }, 45} R_{50}^{-2}
$$

where $L_{\text {ion, } 45}$ is the ionizing luminosity in units of $10^{45}$ $\mathrm{erg} \mathrm{s}^{-1}$ and $R_{50}$ is the distance in units of $50 \mathrm{pc}$.

Typical $\frac{L_{\text {bol }}}{L_{\text {ion }}}$ ratios for luminous Sy1 and QSO1 with $L_{\text {bol }}$ in the range $\sim 10^{45-46} \mathrm{erg} \mathrm{s}^{-1}$ have median value of 3.89 and standard deviation 4.36 . Thus, for a ratio of 3.89 , then $L_{\text {ion,45 }} \sim 2.2$ for MRK 477 . Considering the range of $n$ inferred for each gaseous component, the broad (outflowing), intermediate and narrow kinematic components would be located at $\lesssim 220$ (the upper limit is a consequence of the lower limit on $\left.n \gtrsim 8000 \mathrm{~cm}^{-3}\right), 375 \pm 65$ and $880 \pm 100$ pc respectively. This sets an upper limit on the distance at which the outflow has been originated of $R \lesssim 220$ pc. If densities as high as $\sim 5 \times 10^{5} \mathrm{~cm}^{-3}$ exist in the outflowing gas (Sect. 3.4 .2 ), then $R \lesssim 30$ pc. For comparison, the radio jet extends at $\sim 0.6$ arcsec or $435 \mathrm{pc}$ from the central engine. With all uncertainties involved, it is remarkable that these calculations place the intermediate and narrow components near and beyond the edge of the radio jet respectively. This adds further support to the idea that the radio jet has originated the outflow. In this scenario, our results suggest that the outflow in MRK 477 is concentrated in the nuclear region and does not reach distances beyond $\sim$ few $\times 100 \mathrm{pc}$.

Alternatively, the higher density of the outflowing gas might be explained by the compression exerted on the outflowing gas by the radio-jet induced shocks (Villar Martín et al. 1999; Holt et al. 2011). We note that this scenario is qualitatively different from the scenario where the NLR clouds are compressed by radiation pressure, as assumed in the models used above. The higher density would instead be located at or near the radio jet and decrease moving away from it. In this case a correlation would be expected between the morphology of the radio jet and the ionized gas (whose emission is enhanced due to shock excitation and/or density enhancement). This is actually the case in MRK 477 (Heckman et al. 1997). Both the radio and [O III] images show a bright knot or ridge of emission about $0.4 \operatorname{arcsec}(\sim 290 \mathrm{pc})$ to the northeast of the central source. This clearly shows that the radio jet is interacting with the NLR and enhancing the emission at this location. Thus, an alternate scenario is that this detached knot is responsible for the bulk of emission of the outflowing gas. If so, it must emit a wide range of lines, including $[\mathrm{FeVII}] \lambda 6087$. High spatial resolution spatially extended optical spectroscopy would help discriminate between both scenarios.

\subsection{The origin of the NIR narrow [FeII] $\lambda 1.644$ emission}

We have found that this line is much broader than expected for its critical density and ionization potentials (Sect. 3.2). The underlying broad component has FWHM 4770 \pm 830 $\mathrm{km} \mathrm{s}^{-1}$, larger than any other line and contributes more than half of the total line flux $(\sim 57 \%)$. It also shows the largest $\epsilon=1.3 \pm 0.2$ and is the only line for which the broad component is not blueshifted. All these could be naturally explained in terms of reddening being much weaker in the NIR, so that both the approaching and receding parts of the expanding outflow are observed (unlike the optical lines). However, this is an unlikely explanation since $\mathrm{Pa} \alpha$ shows that the broad component is blueshifted by $-570 \pm 119 \mathrm{~km}$ $\mathrm{s}^{-1}$.

Alternatively, it is possible that $[\mathrm{FeII}] \lambda 1.644$ is relatively enhanced by the shocks induced by the outflow, more than other lines and at a less obscured spatial location (hence the non-blueshift). Outflow induced shocks have been often proposed as a relevant $[\mathrm{FeII}] \lambda 1.644$ excitation mechanism in active galaxies (e.g. Ramos Almeida et al. 2009, Contini et al. ?). As an example, ? studied the kinematics and excitation mechanism of the NIR emission lines in a sample of Seyfert 1 galaxies, including [FeII] $\lambda 1.257$ and $\lambda 1.645 \mu \mathrm{m}$. They found 3 out of 22 sources in which the [FeII] lines were the broadest, even broader than the coronal [SiX] $\lambda 1.252 \mu \mathrm{m}$. They suggest that in these objects [FeII] must arise from and additional source, partially formed in a region distinct from other low-ionization species, which they suggest to be associated with shock excitation from the radio jet. Blietz et al. (1995) also suggest that the [FeII] $\lambda 1.644$ emission in the Sy2 
galaxy NGC1068 is preferentially emitted by gas which is interacting with the nuclear outflow/jet and is possibly located at the interface between the outflow and the dense circumnuclear molecular clouds.

\subsection{Coronal lines}

Detection of coronal emission is confirmed in the NIR spectrum, but not in the optical (Sect. 3.6). In the optical, the highest ionization lines are those emitted by $\mathrm{Fe}^{+6}$, which on the other hand are fainter relative to the low ionization lines than typically found in AGN with strong coronal emission. Coronal lines are generally detected between just a few parsecs and a few hundred parsecs (e.g. Mazzalay et al. 2010 2014). They have been proposed to be formed in a region located at an intermediate distance between the classical NLR and the broad-line region (BLR) (Müller Sánchez et al. 2006). Alternatively, the coronal region might reside in the inner wall of the dusty torus (Murayama \& Taniguchi 1998, Rose et al. 2013) or in the low density surface layer of the radiation pressure confined NLR clouds (S14).

The detection of coronal emission in the NIR and its absence in the optical spectrum of MRK 477 suggests that this region is heavily reddened, partially hidden from our line of sight. Alternatively, since the coronal lines are usually highly nucleated ( $<1 \mathrm{kpc}$ from the nucleus) and much more concentrated than lower ionization NLR features (Rodríguez Ardila et al.2011), it is also possible that they are diluted by the strong continuum contribution within the large aperture of the SDSS fibre (radius $\sim 1 \mathrm{kpc}$ ).

The [SiVI] and [SiX] lines have luminosities 40.0 and 39.8 in $\log$ and $\mathrm{erg} \mathrm{s}^{-1}$ respectively. Rodríguez Ardila et al. (2011) found that these lines display a narrow range in luminosity in Seyfert 1 and 2, with values for most objects located in the interval $\log (L)=39-40$. MRK 477 is at the high end of this range. We measure $[\mathrm{SiVI}] /[\mathrm{SiX}]=1.6 \pm 0.1$, also within the range measured for Seyfert 1 and 2 galaxies by those authors.

\subsection{Detection of narrow optical $\mathrm{Fe}^{+}$emission}

We have identified more than 10 narrow optical $\mathrm{Fe}^{+}$emission lines in the SDSS spectrum of MRK 477. The NIR [FeII] lines at 1.26 and $1.64 \mu \mathrm{m}$ are routinely detected in type 2 Seyferts (e.g. Ramos Almeida, Pérez García \& Acosta-Pulido 2009). However, to our knowledge MRK 477 is the first type 2 AGN with optical $\mathrm{Fe}^{+}$line detections.

To explain the absence of narrow optical $\mathrm{Fe}^{+}$in type 2 AGN, while being prevalent in type 1 objects, Dong et al. (2010) proposed that such emission is confined to a disk-like geometry in the innermost region of the NLR on physical scales of parsecs. This would be smaller than the obscuring torus and within the dust sublimation radius. Iron, which is a refractory element which easily condenses on to dust grains, is in the gaseous phase in the absence of dust (Laor \& Draine 1993). In this scenario the narrow optical $\mathrm{Fe}^{+}$ emitting region is visible along our line of sight in type 1 objects, but obscured by the extent of the dusty torus in type 2 counterparts.

It is however difficult to picture a geometry such as this to explain the optical $\mathrm{Fe}^{+}$emission in MRK 477. Broad permitted hydrogen and helium lines would be expected, unless a BLR did not exist, but this is not the case (Sect. $1)$.

We explore next whether the optical $\mathrm{Fe}^{+}$features can be naturally explained by the intrinsic NLR emission. For this, we compare the measured integrated flux relative to $\mathrm{H} \beta$ of all $\mathrm{Fe}^{+}$lines in the blue (4000-6000 $\AA$ ) and red (6000$7800 \AA$ ) bands with the ratios predicted by photoionization models appropriate for the NLR conditions. We use the radiation-pressure-confined NLR models described in S14. The assumption of these models implies that the line emission is essentially independent of the ionization parameter at the slab surface $U_{0}$, as long as $U_{0} \gg 0.03$ (see Fig. 3 in S14; see also Dopita et al. 2002 and Groves et al. 2004). A continuous distribution of dusty gas as a function of the distance from the nucleus $R$ is considered. This distribution is characterized by $\eta$, the power-law index of the dusty gas covering factor as a function of logarithmic unit of $R$. These types of models are successful in explaining various observations of the NLR (see Dopita et al. 2002 and S14).

We assume solar metallicity, an ionization slope of $\alpha_{\text {ion }}=-1.6$ typical of luminous quasars (Telfer et al.2002), and $\eta=0$, i.e. a constant covering factor per $\log R$, as suggested by the strong line ratios and the IR spectral energy distribution (S14). The photoionization models are calculated using CLOUDY (Ferland et al. 2013), assuming hydrostatic equilibrium. Depletion of refractory elements on to dust grains is taken into account, and the $\mathrm{Fe}^{+}$ion is calculated according to the model described in Verner et al. (1999).

The results for the $\operatorname{FeII}(4000-6000) / \mathrm{H} \beta)$ and $\mathrm{FeII}(6000-7800) / \mathrm{H} \beta)$ are shown in Table 6] considering both dusty and non-dusty models. The uncertainties on the observed ratios account for the errors on the flux measurements and the possible misidentification of some features (Table 1). The models can reproduce successfully both the blue and the red bands. Therefore, the narrow optical $\mathrm{Fe}^{+}$ lines in MRK 477 can be naturally explained by the intrinsic NLR emission due to AGN photoionization. The high luminosity and/or closeness of MRK 477 could be the reason why these lines have been detected, unlike other type 2 AGN.

The effect of dust depletion produces ratios approximately twice lower than those derived from dust-free models. Taking into account the uncertainties, the $\mathrm{Fe}^{+}$band ratios do not discriminate between the two scenarios. Model predictions of lines emitted by other refractory elements such as $\mathrm{Mg}, \mathrm{Ni}$, Ca (also detected for MRK 477, see Sect. 3.5) would help to infer whether the NLR contains dust-free gas. Only the $[\mathrm{CaII}] \lambda 7291$ is predicted by CLOUDY. Calcium is much more sensitive to dust depletion than iron. The dust-free models predict $[\mathrm{Ca}$ II $] \lambda 7291 / \mathrm{H} \beta=0.08$, while the line would be 1000 times fainter (and thus completely undetectable) in the dusty case (see also Villar Martín et al. 1966). The [Ca II] line is tentatively detected with a ratio of $0.02 \pm 0.01$ relative to $\mathrm{H} \beta$ (Fig. 12, third panel). If this detection is confirmed, it would imply that at least a fraction of the NLR gas is dust free. 


\begin{tabular}{|c|c|c|c|c|c|c|c|}
\hline$\left(\frac{\mathrm{FeII}_{4000-6000}}{\mathrm{H} \beta}\right)^{\mathrm{obs}}$ & $\left(\frac{\mathrm{FeII}_{4000-6000}}{\mathrm{H} \beta}\right)^{\mathrm{int}}$ & $\begin{array}{c}(3) \\
\text { Model }_{1} \\
\text { Dust }\end{array}$ & $\begin{array}{c}(4) \\
\text { Model }_{2} \\
\text { Dust-free }\end{array}$ & $\left(\frac{\text { FeII }}{(5)} \frac{(5)-7800}{\mathrm{H} \beta}\right)^{\text {obs }}$ & $\left(\frac{\text { FeII }_{6000-7800}^{(6)}}{\mathrm{H} \beta}\right)^{\text {int }}$ & $\begin{array}{c}\text { Model }_{1} \\
\text { Dust }\end{array}$ & $\begin{array}{c}\text { Model }_{2} \\
\text { Dust-free }\end{array}$ \\
\hline $0.14 \pm 0.03$ & $0.15 \pm 0.1$ & 0.15 & 0.36 & $0.06 \pm 0.03$ & $0.05 \pm 0.04$ & 0.031 & 0.053 \\
\hline
\end{tabular}

Table 6. Comparison between the measured integrated flux relative to $\mathrm{H} \beta$ of all $\mathrm{Fe}^{+}$lines in the blue $(4000-6000 \AA)$ and red (6000-7800 $\AA$ ) bands with the ratios predicted by photoionization models appropriate for the NLR conditions (see the text). The superscripts obs and ${ }^{\text {int }}$ refer to the observed and reddening corrected ratios. Dusty and dust-free model predictions are shown. The narrow optical Fe ${ }^{+}$ lines in MRK 477 can be naturally explained by the intrinsic NLR emission due to AGN photoionization.

\section{CONCLUSIONS}

We perform a detailed spectral analysis and characterization of the NLR of the nearest obscured quasar (QSO2) MRK 477 at $z=0.037$ based on the SDSS optical and NIR H+K spectra obtained with WHT-LIRIS.

(i) We confirm the hybrid nature $(\mathrm{AGN}+$ starburst) proposed by Heckman et al. 1997, based on the new detection of the "red bump" at $~ 5800 \AA$ due to WR stars, which implies that the system has undergone recent star formation.

(ii) The optical spectrum of MRK 477 is rich in emission lines, with $\sim 90$ detected features. In spite of the lack of spatial information of the SDSS spectrum, the spectral decomposition of numerous lines has allowed us to characterize, at least partially, the spatial structure and the gradients in the physical and kinematic properties of the gas.

(iii) Gas densities within the range $\sim 1945_{-460}^{+670}$ and up to $>10^{4} \mathrm{~cm}^{-3}$ are confirmed in the NLR of MRK 477 .

(iv) As in many other active galaxies (AGN), a significant correlation is found between the lines FWHM and the critical density $\log \left(n_{\text {crit }}\right)$. We propose that this correlation is caused by the outflow and its impact on the gas kinematics. This could be the case in other AGNs.

(v) MRK 477 is an example of a radio quiet powerful AGN where negative feedback (the nuclear outflow) can be dominated by the radio structures. The outflow emission has been isolated in many emission lines covering a large range of ionization potentials and critical densities (from [OI] 26300 to $[\mathrm{FeVII}] \lambda 6086)$. The outflowing gas, which is concentrated within $\mathrm{R} \sim$ few $\times 100 \mathrm{pc}$ from the central engine, is $>13$ times denser $\left(n \gtrsim 8,000 \mathrm{~cm}^{-3}\right)$ than the ambient non perturbed gas $\left(n \sim 400-630 \mathrm{~cm}^{-3}\right)$. Marginal evidence is found for densities as high as $n \gtrsim 5 \times 10^{5} \mathrm{~cm}^{-3}$ in the outflowing gas. It is possible that the density enhancement is due to the gas compression produced by jet induced shocks. This is supported by the correlation between the radio and [OIII] $\lambda 5007$ morphologies found by Heckman et al. (1997).

Alternatively, the density enhancement is not related to the jet. Instead, it might be a reflection of the NLR intrinsic density gradient, consequence of the gas being compressed by radiation pressure. In this scenario, and based on the comparative study between the density and the kinematic properties of the outflowing and the ambient gas, we conclude that the outflow has been generated at $\lesssim 220$ pc (possibly at $\lesssim 30 \mathrm{pc}$ ) from the AGN. We find evidence of how its effects weaken as it propagates outwards, following the NLR density gradient. Beyond the radio jet edge, the gas emission is dominated by ambient less dense, non perturbed gas. This adds further support to the idea that the radio jet has triggered the outflow. (vi) The $[\mathrm{FeII}] \lambda 1.644 \mu \mathrm{m}$ line presents a very different behaviour than the rest of the emission lines. It shows the most extreme effects of the outflow, with an underlying broad component of $\mathrm{FWHM}=4770 \pm 830 \mathrm{~km} \mathrm{~s}^{-1}$. Its properties suggest that its emission is enhanced by shocks induced by the nuclear outflow/jet and is preferentially emitted at a different, less reddened spatial location, maybe the interface between the outflow and the dense circumnuclear molecular clouds.

(vii) More than 10 narrow optical $\mathrm{Fe}^{+}$emission lines have been detected in the SDSS spectrum of MRK 477. To our knowledge, this is the first type 2 AGN with such a detection. We show that these lines can be explained as the natural emission from NLR gas photoionized by the AGN. Emission lines associated with other highly refractory elements $(\mathrm{Mg}$, possibly $\mathrm{Ni})$ are also detected. If the tentative detection of the [Ca II] $\lambda 7291$ line is confirmed, this would imply that at least part of the NLR gas is dust-free.

(viii) Coronal line emission is confirmed in the NIR, but not in the optical SDSS spectrum. The coronal region might be heavily reddened, partially hidden from our line of sight. Alternatively its optical emission might be diluted due to the large SDSS fibre aperture. The coronal region also participates in the outflow.

(ix) $\mathrm{Pa} \alpha$ is spatially extended along the radio and [OIII] emission axes, up to a maximum radial extension of $\sim 1.5 \mathrm{kpc}$ from the AGN.

\section{ACKNOWLEDGMENTS}

Thanks to an anonymous referee for useful comments on the paper.

This work has been funded with support from the Spanish Ministerio de Economía y Competitividad through the grant AYA2012-32295. JS acknowledges financial support from the Alexander von Humboldt foundation. CRA is supported by a Marie Curie Intra European Fellowship within the 7th European Community Framework Programme (PIEF-GA-2012-327934). RGD acknowledges support through the grant AYA2010-15081.

This work is partially based on data obtained as part of the WHT service programme. The WHT and its service programme are operated on the island of La Palma by the Isaac Newton Group in the Spanish Observatorio del Roque de los Muchachos of the Instituto de Astrofísica de Canarias. We thank the WHT staff for performing the observations.

The work is also based on data from Sloan Digital Sky Survey. Funding for the SDSS and SDSS-II has been provided by the Alfred P. Sloan Foundation, the Participating 
Institutions, the National Science Foundation, the U.S. Department of Energy, the National Aeronautics and Space Administration, the Japanese Monbukagakusho, the Max Planck Society, and the Higher Education Funding Council for England. The SDSS website is http://www.sdss.org/

The SDSS is managed by the Astrophysical Research Consortium for the Participating Institutions. The Participating Institutions are the American Museum of Natural History, Astrophysical Institute Potsdam, University of Basel, University of Cambridge, Case Western Reserve University, University of Chicago, Drexel University, Fermilab, the Institute for Advanced Study, the Japan Participation Group, Johns Hopkins University, the Joint Institute for Nuclear Astrophysics, the Kavli Institute for Particle Astrophysics and Cosmology, the Korean Scientist Group, the Chinese Academy of Sciences (LAMOST), Los Alamos National Laboratory, the Max-Planck-Institute for Astronomy (MPIA), the Max-Planck-Institute for Astrophysics (MPA), New Mexico State University, Ohio State University, University of Pittsburgh, University of Portsmouth, Princeton University, the United States Naval Observatory, and the University of Washington.

\section{REFERENCES}

Acosta Pulido J. et al. ING Newsl., 7, 15

Appenzeller I., Oestreicher R., 1988, AJ, 95, 45

Bennert N., Jungwiert B., Komossa S., Haas M., Chini R., 2006, A\&A, 456, 953

Bennert N., Jungwiert B., Komossa S., Haas M., Chini R., 2006, A\&A, 459, 55

Binette L., Wang J., Villar-Martín M., Martin P., Magris G., 1993, ApJ, 414, 535

Blietz M., Cameron M., Drapatz S., Genzel R., Krabbe A., van der Werf P., Sternberg A., Ward M., 1994, ApJ, 421, 92

De Robertis M.M., Ostrbrock D.E., 1984, ApJ, 286, 171, 953

De Robertis M.M., Ostrbrock D.E., 1986, ApJ, 301, 727

De Robertis M.M., 1987, ApJ, 316, 597

Dong X. B., Ho L., Wang J.G., Want T.G., Wang H., Xiaohui F., Hongyan Z., 2010, ApJL, 721, L143

Dopita M. A., Groves B. A., Sutherland R. S., Binette L., Cecil G., 2002, ApJ, 572, 753

Dougados C., Bacciotti F., Cabrit S., Nisini B., 2010, in Jets from Young Stars IV, Lecture Notes in Physics, Volume 793. ISBN 978-3-642-02290-6. Springer-Verlag Berlin Heidelberg, 2010, p. 213

Espey B., 1994, in Shlosman I., ed., Proc. Conf. MassTransfer Induced Activity in Galaxies. Cambridge Univ. Press, Cambrdige, p.121

Falcón-Barroso J., Ramos Almeida C., Böker T., Schinnerer E., Knapen J. H., Lancon A., Ryde, S., 2014, MNRAS, 438, 329

Ferland G. J. et al., 2013, Rev. Mex. Astron. Astrofis., 49, 137

González Delgado R., Heckman T., Leitherer C., Meurer G., Krolik J., Wilson A., Kinney A., Koratkar A., 1998, ApJ, 505, 174

Groves B. A., Dopita M. A., Sutherland R. S., 2004, ApJS, 153,75
Guseva N., Izotov Y., Thuan T., 2000, ApJ, 531, 776

Heckman T. M., González Delgado R., Leitherer C., Meurer G.R., Krolik J., Wilson A.S., Koratkar A., Kinney A., 1997, ApJ, 482, 114

Holt J., Tadhunter C. N., Morganti R., Emonts B., 2011, MNRAS, 410, 1527

Keenan F. P., Aller L. H., Bell K. L., Hyung S., McKenna F. C., Ramsbottom C. A., 1996, MNRAS, 271,1073

Koss M., Mushotzky R., Treister E., Veilleux S., Vasudevan R., Trippe M., 2012, ApJ, 746, 22

Krug H., Rupke D., Veilleux S., 2010, ApJ, 708, 1145

Lamastra A., Bianchi S., Matt G., Perola G.C., Barcons X., Carrera F.J., 2009, A\&A, 504, 73

Laor A., Draine B., 1993, ApJ, 402, 441

Luridiana V., Morisset C., Shaw R., 2015, A\&A, 573, 42

Manchado A., et al. 2004, Proc. SPIE, 5492, 1094

Mazzalay X., Rodríguez-Ardila A., Komossa S., 2010, MNRAS, 405, 1315

Mazzalay X. et al. 2013, MNRAS, 428, 2389

Mazzalay X. et al., 2014, MNRAS, 438, 2036

Müller Sánchez F., Davies R. I., Eisenhauer F., Tacconi L.

J., Genzel R., Sternberg A., 2006, A\&A, 454, 481

Müller Sánchez F., Prieto M. A., Hicks E. K. S., VivesArias H., Davies R. I., Malkan M., Tacconi L. J., Genzel R., 2011, ApJ, 739, 69

Murayama T., Taniguchi Y., 1998, ApJ, 503, L115

Osterbrock D.E., 1989, Astrophysics of Gaseous Nebulae and Active Galactic Nuclei. University Science Books, Mill Valley, CA

Penston M.V., Fosbury R.A.E., Boksenberg A., Ward M.J., Wilson A.S., 1984, 208, 347

Pier E., Voit G.M., 1994, ApJ, 450, 628

Ramos Almeida C., Prez Garca A. M., Acosta-Pulido J. A., 2009, AJ, 137, 179

Rodríguez Ardila A., Prieto M.A., Viegas S., Grwenland R., 2006, ApJ, 653, 109

Rodríguez Ardila A., Pastoriza M. G., Viegas S., Sigut T. A., Pradhan A. K., 2004, A\&A, 425, 457

Rodríguez Ardila A., Prieto M.A., Portilla J.G., Tejeiro J.M., 2011, ApJ, 743, 100

Rose M., Tadhunter C. N., Holt J., Rodríguez Zaurín J., 2013, MNRAS, 432, 2150

Schaerer D., Vacca W. D. W., 1998, ApJ, 497, 618

Shen Y. et al., 2011, ApJS, 194, 45

Shu X., Wang J., Jiang P., Fan L., Wang T., 2007, ApJ, 657,167

Shuder J., Osterbrock D., 1981, ApJ, 250, 55

Stern J., Laor A., 2012, MNRAS, 426, 2703

Stern J., Laor A., Baskin A., 2014, MNRAS, 438, 901 (S14)

Telfer R. C., Zheng W., Kriss G. A., Davidsen A. F., 2002, ApJ, 565, 773

Tran H., Miller J., Kay L., 1992, ApJ, 397, 452

Tran H., 1995, ApJ, 440, 578

Veilleux S., 1988, AJ, 95, 1695

Verón-Cetty M.P., Joly M., Véron P., 2004, A\&A, 417, 515

Verón-Cetty M.P., Véron P., Joly M., Kollatschny W., 2013, A\&A, 475, 487

Verner E. M., Verner D. A., Korista K. T., Ferguson J. W., Hamann F., Ferland G. J., 1999, ApJS, 120, 101

Villar Martín M., Binette L., 1996, A\&A, 309, 97

Villar Martín M., Tadhunter C., Morganti R., Axon D., Koekemoer A., 1999, MNRAS, 307, 24 
Villar Martín M., Emonts B., Humphrey A., Cabrera Lavers A., Binette L., 2014, MNRAS, 440, 3202

Whittle M., 1992, ApJS, 79, 49

Wright E. L., 2006, PASP, 118, 1711

York D. G. et al., 2000, AJ, 120, 1579

Zakamska N. L. et al. 2003, AJ, 126, 2125

Zhang S.H., Bian W.H., Huang K.L., 2008, MNRAS, 488, 113

Zwicky F., 1966, ApJ, 143, 192 